\title{
Nonlinear Dynamic Analysis of Macrofiber Composites Laminated Shells
}

\author{
Xiangying Guo, ${ }^{1}$ Dameng Liu, ${ }^{1}$ Wei Zhang, ${ }^{1}$ Lin Sun, ${ }^{2}$ and Shuping Chen ${ }^{3}$ \\ ${ }^{1}$ Beijing Key Laboratory of Nonlinear Vibrations and Strength of Mechanical Structures, College of Mechanical Engineering, \\ Beijing University of Technology, Beijing 100124, China \\ ${ }^{2}$ CSIC 760 Institute, Dalian, China \\ ${ }^{3}$ College of Mathematics, Xiamen University of Technology, Xiamen 361024, China
}

Correspondence should be addressed to Wei Zhang; sandyzhang0@yahoo.com

Received 16 November 2016; Accepted 26 March 2017; Published 9 May 2017

Academic Editor: Zhengong Zhou

Copyright (C) 2017 Xiangying Guo et al. This is an open access article distributed under the Creative Commons Attribution License, which permits unrestricted use, distribution, and reproduction in any medium, provided the original work is properly cited.

\begin{abstract}
This work presents the nonlinear dynamical analysis of a multilayer $d_{31}$ piezoelectric macrofiber composite (MFC) laminated shell. The effects of transverse excitations and piezoelectric properties on the dynamic stability of the structure are studied. Firstly, the nonlinear dynamic models of the MFC laminated shell are established. Based on known selected geometrical and material properties of its constituents, the electric field of MFC is presented. The vibration mode-shape functions are obtained according to the boundary conditions, and then the Galerkin method is employed to transform partial differential equations into two nonlinear ordinary differential equations. Next, the effects of the transverse excitations on the nonlinear vibration of MFC laminated shells are analyzed in numerical simulation and moderating effects of piezoelectric coefficients on the stability of the system are also presented here. Bifurcation diagram, two-dimensional and three-dimensional phase portraits, waveforms phases, and Poincare diagrams are shown to find different kinds of periodic and chaotic motions of MFC shells. The results indicate that piezoelectric parameters have strong effects on the vibration control of the MFC laminated shell.
\end{abstract}

\section{Introduction}

MFC is a piezoelectric fiber material, consisting of monotonic piezoelectric material, epoxy matrix, and electrodes with a specific arrangement, which can be considered as homogenized orthotropic materials with arbitrary piezoelectric fiber angles like composite structures.

There are generally three types of piezoelectric material which researches focus on mainly. The first type of the piezoelectric fiber composite material is produced by Smart Material Corp, treated as 1-3 composite. The second type is originally developed by MIT, named active fiber composite actuators. Macrofiber composite (MFC) is referred to as the third type presented by NASA Langley Research Center. Since several applications require conformable and packaged piezoelectric structures or actuators, MFC material become widely applied in both academic and industrial field, which would be used for an easier integration in smart, intelligent, or adaptive structures [1-3]. Great advantages of the MFC material, which is able to respond to changing environment and controlling structural deformation, have led to a new generation for aerospace structures especially for morphing aircrafts [4].

Theoretical analyses are the basis to obtain dynamical characteristics of MFC material subjected to different excitations. In some researches, piezoelectric characteristics of MFC are studied by nonlinear constitutive equations and numerical simulation, including higher-order terms and corresponding coefficients [5-7] or by using strain dependent or effective piezoelectric coefficients in constitutive equations [8-11].

Recently, different finite element models are developed to analyze the nonlinear dynamical behavior of MFC laminated materials [12-16]. Gohari et al. [17] present an analytical solution to obtain static deformation and optimal shape control of smart laminated cantilever piezoelectric composite 
hybrid plates and beams under several coupled loads using piezoelectric actuators. Park and Kim [18] show that the aerothermal large deflection of the composite panel can be suppressed using MFC actuators and the excessive actuation of the MFC can cause snap-through phenomena. Kim et al. [19] study the vibration suppression of an end-capped cylindrical shell structure with surface bonded macrofiber composite actuators and analyze the dynamic characteristics of the cylindrical shell structure.

Dano and Jullière [20] investigate the use of macrofiber composite (MFC) actuators to actively control thermally induced deformations in composite structures. Korayem and Homayooni [21] analyze the influence of the applied voltage on vibration frequency of a multilayer piezoelectric microplate as well as the size effects on the macroplate at different boundary conditions. Li et al. [22] present the active control of random vibration for piezoelectric fiber reinforced composites laminated plates and discuss the effect of piezoelectric fiber orientation in the PFRC layers. Suresh Kumar and Ray [23] analyze the geometrically nonlinear vibrations of doubly curved smart sandwich shells integrated with a patch of 1-3 piezoelectric composites active constrained layer damping (ACLD) treatment.

Experimental studies are effective in verifying theoretical results. The effective properties of piezoelectric composites on the interface material are reported by using theoretical and numerical methods in [24, 25]. Andrianov et al. [26] focus on the evaluation of the homogeneous properties of the active layers in both $d_{31}$ and $d_{33}$ MFCs. Biscani et al. [27] study the effective electromechanical properties of MFC transducers and compare the material properties of the system with experimental data. Prasath and Arockiarajan $[28,29]$ present total packing effects on the overall properties of MFC using experimental models. Based on the developed Kirchhoff plate theory, in some articles [30, 31], the actuation responses of $d_{33}$ and $d_{31}$ MFCs integrated smart structures are studied subjected to transverse excitations. Kashiwao et al. [32] propose an optimization method of a vibration energy harvesting system which is made of MFC piezoelectric elements.

\section{Mechanical Model}

MFC mainly consist of piezoceramic fibers, epoxy matrix, and electrodes, which have two different types of structures, named $d_{31}$ and $d_{33}$ modes. Here, a $d_{31}$ MFC middling thick cross-ply laminated shell is considered, where the piezomacrofiber is polarized in the thickness direction and fully embedded in the epoxy matrix as shown in Figure 1. The shell is a cantilever doubly curved cylindrical shell with a rectangular base. The orthogonal curvilinear coordinate is shown in Figure 2, and $\alpha$ and $\beta$ curves are along the lines of curvatures on the middle surface and $\gamma$ is perpendicular to the middle surface of the shell. Geometric dimensions of the shell in the curvilinear coordinate are the lengths $a$ and $b$, and the thickness $h$, and the principal radii of the curvatures $R_{1}$ and $R_{2}$. The displacements of an arbitrary point within the shell in the coordinate $(\alpha, \beta, \gamma)$ are denoted with $u, v$, and $w$, respectively. $w$ is taken as positive vector going outward

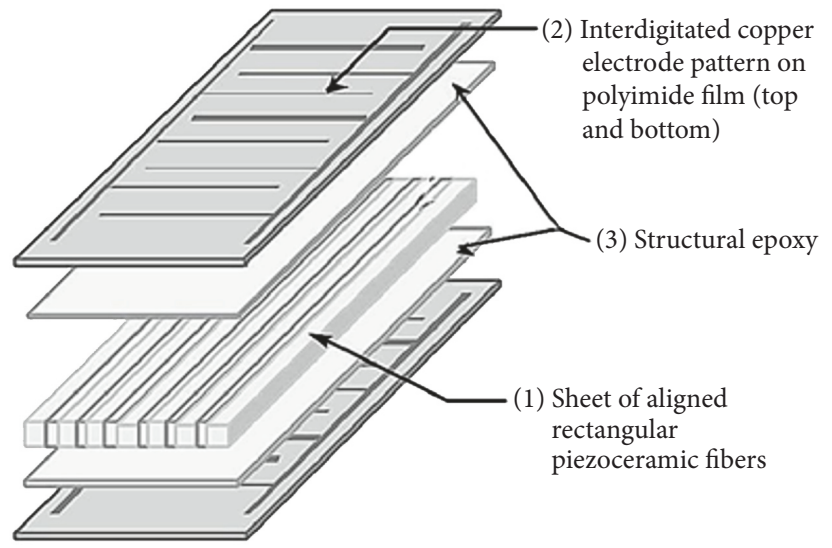

Figure 1: MFC structure.

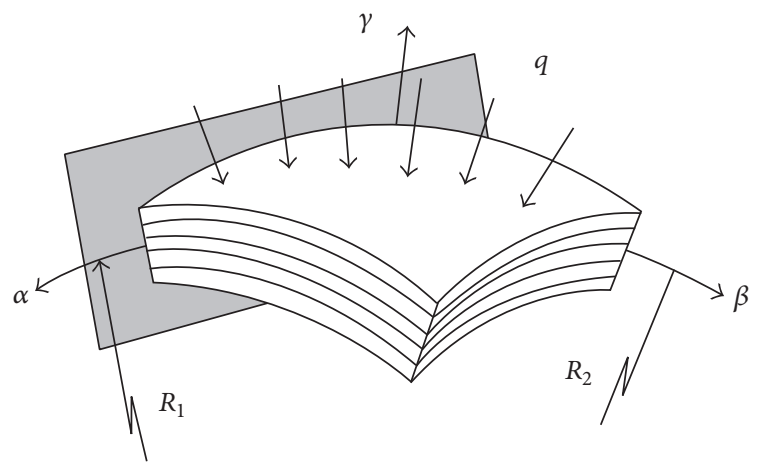

FIgURE 2: The model of MFC shell.

from the center of the smallest radius of the curvature. The shell is subjected to a transverse excitation given as $q=f_{0}+$ $f_{1} \cos (\Omega t)$, as shown in Figure 2 .

According to Reddy's third-order theory, the displacement fields at an arbitrary point in the composite shell are given in the following form:

$$
\begin{aligned}
& u=u_{0}+\gamma \phi_{1}-\frac{4}{3 h^{2}} \gamma^{3}\left(\phi_{1}+\frac{\partial w_{0}}{\partial \alpha}\right), \\
& v=v_{0}+\gamma \phi_{2}-\frac{4}{3 h^{2}} \gamma^{3}\left(\phi_{2}+\frac{\partial w_{0}}{\partial \beta}\right), \\
& w=w_{0},
\end{aligned}
$$

where $u_{0}, v_{0}$, and $w_{0}$ are the original displacements at the mid-plane of the MFC shell in the $\alpha, \beta, \gamma$ directions; $\phi_{1}$ and $\phi_{2}$ represent the rotations of transverse normal at the mid-plane about the $\alpha$ and $\beta$ axes. 
Using von Karman's geometric relationship, the strain and displacements of the MFC shell can be written as follows:

$$
\begin{aligned}
& \varepsilon_{1}=\frac{1}{A_{1}}\left(\frac{\partial u}{\partial \alpha}+\frac{1}{a_{2}} \frac{\partial a_{1}}{\partial \beta} v+\frac{a_{1}}{R_{1}} w\right), \\
& \varepsilon_{2}=\frac{1}{A_{2}}\left(\frac{\partial v}{\partial \beta}+\frac{1}{a_{1}} \frac{\partial a_{2}}{\partial \alpha} v+\frac{a_{2}}{R_{2}} w\right), \\
& \varepsilon_{3}=\frac{\partial w}{\partial \gamma} \\
& \varepsilon_{4}=\frac{1}{A_{2}} \frac{\partial w}{\partial \beta}+A_{2} \frac{\partial}{\partial \gamma}\left(\frac{v}{A_{2}}\right), \\
& \varepsilon_{5}=\frac{1}{A_{1}} \frac{\partial w}{\partial \alpha}+A_{1} \frac{\partial}{\partial \gamma}\left(\frac{u}{A_{1}}\right), \\
& \varepsilon_{6}=\frac{A_{2}}{A_{1}} \frac{\partial}{\partial \alpha}\left(\frac{v}{A_{2}}\right)+\frac{A_{1}}{A_{2}} \frac{\partial}{\partial \beta}\left(\frac{u}{A_{1}}\right) .
\end{aligned}
$$

Here, $A_{1}, A_{2}$ are the Lame coefficients of the shell and can be expressed as $A_{1}=a_{1}\left(1+\gamma / R_{1}\right), A_{2}=a_{2}\left(1+\gamma / R_{2}\right)$.

The stress-strain relationships for the MFC material are given by

$$
\begin{aligned}
& \sigma_{p}=C_{p q} \varepsilon_{q}-e_{k p} E_{k}, \\
& D_{i}=e_{i q} \varepsilon_{q}+k_{i k} E_{k},
\end{aligned}
$$

where $E_{k}$ is the electric field intensity and $e_{k p}$ represents the piezoelectric constant.

All piezoelectric fibers are considered to be poled in $\gamma$ (through-thickness) direction. Therefore, it can be assumed that in-plane electric fields vanish (i.e., $E_{1}=E_{2}=0$ ), and the following reduced constitutive equations hold:

$$
\begin{aligned}
& \left\{\begin{array}{l}
\sigma_{1} \\
\sigma_{2} \\
\sigma_{3} \\
\sigma_{4} \\
\sigma_{5} \\
\sigma_{6}
\end{array}\right\} \\
& =\left[\begin{array}{ccccccc}
C_{11} & C_{12} & C_{13} & 0 & 0 & 0 \\
C_{12} & C_{11} & C_{13} & 0 & 0 & 0 \\
C_{13} & C_{13} & C_{13} & 0 & 0 & 0 \\
0 & 0 & 0 & C_{44} & 0 & 0 \\
0 & 0 & 0 & 0 & C_{44} & 0 \\
0 & 0 & 0 & 0 & 0 & \frac{\left(C_{11}-C_{12}\right)}{2}
\end{array}\right]\left\{\begin{array}{l}
\varepsilon_{1} \\
\varepsilon_{2} \\
\varepsilon_{3} \\
\varepsilon_{4} \\
\varepsilon_{5} \\
\varepsilon_{6}
\end{array}\right\}
\end{aligned}
$$

$$
+\left[\begin{array}{ccc}
0 & 0 & -e_{31} \\
0 & 0 & -e_{31} \\
0 & 0 & -e_{33} \\
0 & -e_{15} & 0 \\
-e_{15} & 0 & 0 \\
0 & 0 & 0
\end{array}\right]\left\{\begin{array}{l}
E_{1} \\
E_{2} \\
E_{3}
\end{array}\right\},
$$

where $C_{11}=E_{11} /\left(1-v_{12} v_{21}\right), C_{12}=v_{12} E_{22} /\left(1-v_{12} v_{21}\right)$, and $C_{44}=G_{23}, C_{66}=G_{12}, E_{i i}$ are the stiffness modulus of the MFC shell, $v_{i j}$ denote Poisson's ratio, and $G_{i j}$ represent the shear modulus.

The stiffness elements of the symmetric cross-ply composite laminated shell are expressed in terms of the stiffness coefficients as follows:

$$
\begin{aligned}
& \left(A_{i j}, B_{i j}, D_{i j}, E_{i j}, F_{i j}, H_{i j}\right) \\
& \quad=\sum_{k=1}^{N} \int_{\gamma_{k}}^{\gamma_{k+1}} C_{i j}^{k}\left(1, \gamma, \gamma^{2}, \gamma^{3}, \gamma^{4}, \gamma^{6}\right) d \gamma,
\end{aligned}
$$

$$
(i, j=1,2,6)
$$

$$
\left(A_{i j}, D_{i j}, F_{i j}\right)=\sum_{k=1}^{N} \int_{\gamma_{k}}^{\gamma_{k+1}} C_{i j}^{k}\left(1, \gamma^{2}, \gamma^{4}\right) d \gamma,
$$

$$
(i, j=4,5)
$$

$$
I_{i}=\int_{-h / 2}^{h / 2} \rho \gamma^{i} d \gamma, \quad(i=0,1,2,3,4,6)
$$

The relationship between curvilinear coordinate system and rectangular coordinate system is $d x=a_{1} d \alpha, d y=a_{2} d \beta$, $d z=d \gamma$. Substitute these transformations into (1a), (1b), and (1c) and then apply Hamilton's principle; the nonlinear governing equations of motion in terms of generalized displacements for the MFC shell can be obtained as follows:

$$
\begin{aligned}
& a_{11} \frac{\partial^{2} u_{0}}{\partial x^{2}}+a_{12} \frac{\partial^{2} u_{0}}{\partial y^{2}}+a_{13} \frac{\partial^{2} v_{0}}{\partial x \partial y}+a_{14} \frac{\partial u_{0}}{\partial x}+a_{15} \frac{\partial v_{0}}{\partial x} \\
& -a_{16} \frac{\partial u_{0}}{\partial y}-a_{17} \frac{\partial v_{0}}{\partial y}-a_{18} \frac{\partial^{3} w_{0}}{\partial x^{2}}-a_{19} \frac{\partial^{3} w_{0}}{\partial x \partial y^{2}} \\
& -a_{20} \frac{\partial^{2} w_{0}}{\partial x^{2}}+a_{21} \frac{\partial^{2} w_{0}}{\partial y^{2}}-a_{22} \frac{\partial^{2} w_{0}}{\partial x \partial y}+a_{23} \frac{\partial w_{0}}{\partial x} \\
& +a_{24} \frac{\partial^{2} \phi_{1}}{\partial x^{2}}+a_{25} \frac{\partial^{2} \phi_{1}}{\partial y^{2}}+a_{26} \frac{\partial^{2} \phi_{2}}{\partial x \partial y}+a_{27} \frac{\partial \phi_{1}}{\partial x} \\
& +a_{28} \frac{\partial \phi_{2}}{\partial x}+a_{29} \frac{\partial \phi_{1}}{\partial y}+a_{30} \frac{\partial \phi_{2}}{\partial y}+a_{31} u_{0} \\
& +a_{32} \phi_{1}=I_{0} \ddot{u}_{0},
\end{aligned}
$$




$$
\begin{aligned}
& b_{11} \frac{\partial^{2} v_{0}}{\partial y^{2}}+b_{12} \frac{\partial^{2} v_{0}}{\partial x^{2}}+b_{13} \frac{\partial^{2} u_{0}}{\partial x \partial y}+b_{14} \frac{\partial v_{0}}{\partial y}+b_{15} \frac{\partial u_{0}}{\partial y} \\
& -b_{16} \frac{\partial v_{0}}{\partial x}-b_{17} \frac{\partial u_{0}}{\partial x}-b_{18} \frac{\partial^{3} w_{0}}{\partial y^{3}}-b_{19} \frac{\partial^{3} w_{0}}{\partial x^{2} \partial y} \\
& -b_{20} \frac{\partial^{2} w_{0}}{\partial y^{2}}+b_{21} \frac{\partial^{2} w_{0}}{\partial x^{2}}-b_{22} \frac{\partial^{2} w_{0}}{\partial x \partial y}+b_{23} \frac{\partial w_{0}}{\partial y} \\
& +b_{24} \frac{\partial^{2} \phi_{2}}{\partial y^{2}}+b_{25} \frac{\partial^{2} \phi_{2}}{\partial x^{2}}+b_{26} \frac{\partial^{2} \phi_{1}}{\partial x \partial y}+b_{27} \frac{\partial \phi_{2}}{\partial y} \\
& +b_{28} \frac{\partial \phi_{1}}{\partial y}+b_{29} \frac{\partial \phi_{1}}{\partial y}+b_{30} \frac{\partial \phi_{1}}{\partial x}+b_{31} v_{0} \\
& +b_{32} \phi_{2}=I_{0} \ddot{v}_{0} \\
& -c_{11} \frac{\partial^{4} w_{0}}{\partial x^{4}}-c_{12} \frac{\partial^{4} w_{0}}{\partial y^{4}}-c_{13} \frac{\partial^{3} w_{0}}{\partial x^{3}}-c_{14} \frac{\partial^{3} w_{0}}{\partial y^{3}} \\
& -c_{15} \frac{\partial^{4} w_{0}}{\partial x^{2} \partial y^{2}}+c_{16} \frac{\partial^{3} w_{0}}{\partial x^{2} \partial y}+c_{17} \frac{\partial^{3} w_{0}}{\partial x \partial y^{2}} \\
& +c_{18}\left(\frac{\partial w_{0}}{\partial x}\right)^{2}+c_{19} \frac{\partial^{2} w_{0}}{\partial x^{2}}+c_{20}\left(\frac{\partial w_{0}}{\partial y}\right)^{2} \\
& +c_{21} \frac{\partial^{2} w_{0}}{\partial y^{2}}+c_{22} \frac{\partial u_{0}}{\partial x}+c_{23} \frac{\partial v_{0}}{\partial y}+c_{24} \frac{\partial u_{0}}{\partial x} \frac{\partial^{2} w_{0}}{\partial x^{2}} \\
& +c_{25} \frac{\partial^{2} u_{0}}{\partial x^{2}} \frac{\partial w_{0}}{\partial x}+c_{26} v_{0} \frac{\partial^{2} w_{0}}{\partial x^{2}}+c_{27} \frac{\partial v_{0}}{\partial x} \frac{\partial w_{0}}{\partial x} \\
& +c_{28} w_{0} \frac{\partial^{2} w_{0}}{\partial x^{2}}+c_{29} \frac{\partial v_{0}}{\partial y} \frac{\partial^{2} w_{0}}{\partial x^{2}}+c_{30} u_{0} \frac{\partial^{2} w_{0}}{\partial x^{2}} \\
& +c_{31} \frac{\partial u_{0}}{\partial x} \frac{\partial w_{0}}{\partial x}+c_{32} \frac{\partial v_{0}}{\partial x} \frac{\partial^{2} w_{0}}{\partial x \partial y}+c_{33} \frac{\partial^{2} v_{0}}{\partial x^{2}} \frac{\partial w_{0}}{\partial y} \\
& -c_{34} u_{0} \frac{\partial^{2} w_{0}}{\partial x \partial y}-c_{35} \frac{\partial u_{0}}{\partial x} \frac{\partial w_{0}}{\partial y}+c_{36} \frac{\partial u_{0}}{\partial y} \frac{\partial^{2} w_{0}}{\partial x \partial y} \\
& +c_{37} \frac{\partial^{2} u_{0}}{\partial x \partial y} \frac{\partial w_{0}}{\partial y}-c_{38} v_{0} \frac{\partial^{2} w_{0}}{\partial x \partial y}-c_{39} \frac{\partial v_{0}}{\partial x} \frac{\partial w_{0}}{\partial y} \\
& +c_{56} \frac{\partial^{2} \phi_{2}}{\partial x^{2}}+c_{57} \frac{\partial^{2} \phi_{1}}{\partial x^{2}}+c_{58} \frac{\partial^{2} \phi_{2}}{\partial y^{2}}+c_{59} \frac{\partial^{2} \phi_{1}}{\partial y^{2}} \\
& +c_{60} \frac{\partial^{2} \phi_{1}}{\partial x \partial y}+c_{61} \frac{\partial^{2} \phi_{2}}{\partial x \partial y}-c_{62} u_{0}-c_{63} v_{0}-c_{64} w_{0} \\
& +f \cos (\Omega t)-k \dot{w}_{0}=c_{1} I_{4}\left(\frac{\partial \ddot{\phi}_{1}}{\partial x}+\frac{\partial \ddot{\phi}_{2}}{\partial y}\right) \\
& -c_{1}^{2} I_{6}\left[\left(\frac{\partial \ddot{\phi}_{1}}{\partial x}+\frac{\partial \ddot{\phi}_{2}}{\partial y}\right)+\left(\frac{\partial^{2} \ddot{w}_{0}}{\partial x^{2}}+\frac{\partial^{2} \ddot{w}_{0}}{\partial y^{2}}\right)\right] \\
& +I_{0} \ddot{w}_{0} \text {, } \\
& d_{11} \frac{\partial^{2} \phi_{1}}{\partial x^{2}}+d_{12} \frac{\partial^{2} \phi_{1}}{\partial y^{2}}+d_{13} \frac{\partial \phi_{1}}{\partial x}+d_{14} \frac{\partial \phi_{1}}{\partial y}+d_{15} \frac{\partial \phi_{2}}{\partial x} \\
& +d_{16} \frac{\partial \phi_{2}}{\partial y}+d_{17} \frac{\partial^{2} \phi_{2}}{\partial x \partial y}+d_{18} \frac{\partial^{3} w_{0}}{\partial x^{3}}+d_{19} \frac{\partial^{3} w_{0}}{\partial x \partial y^{2}} \\
& +d_{20} \frac{\partial^{2} w_{0}}{\partial x^{2}}+d_{21} \frac{\partial^{2} w_{0}}{\partial y^{2}}+d_{22} \frac{\partial^{2} w_{0}}{\partial x \partial y}+d_{23} \frac{\partial w_{0}}{\partial x} \\
& +d_{24} u_{0}+d_{25} \phi_{1}=I_{2} \ddot{\phi}_{1}-c_{1} I_{4} \ddot{\phi}_{1}-c_{1} I_{4} \frac{\partial \ddot{w}_{0}}{\partial x} \\
& +c_{1}\left(-I_{4} \ddot{\phi}_{1}-c_{1} I_{6} \ddot{\phi}_{1}+c_{1} I_{6} \frac{\partial \ddot{w}_{0}}{\partial x}\right), \\
& e_{11} \frac{\partial^{2} \phi_{2}}{\partial y^{2}}+e_{12} \frac{\partial^{2} \phi_{2}}{\partial x^{2}}+e_{13} \frac{\partial \phi_{2}}{\partial y}+e_{14} \frac{\partial \phi_{2}}{\partial x}+e_{15} \frac{\partial \phi_{1}}{\partial y} \\
& +e_{16} \frac{\partial \phi_{1}}{\partial x}+e_{17} \frac{\partial^{2} \phi_{1}}{\partial x \partial y}+e_{18} \frac{\partial^{3} w_{0}}{\partial y^{3}}+e_{19} \frac{\partial^{3} w_{0}}{\partial x^{2} \partial y} \\
& +e_{20} \frac{\partial^{2} w_{0}}{\partial y^{2}}+e_{21} \frac{\partial^{2} w_{0}}{\partial x^{2}}+e_{22} \frac{\partial^{2} w_{0}}{\partial x \partial y}+e_{23} \frac{\partial w_{0}}{\partial y} \\
& +e_{24} v_{0}+e_{25} \phi_{2}=I_{2} \ddot{\phi}_{2}-c_{1} I_{4} \ddot{\phi}_{2}-c_{1} I_{4} \frac{\partial \ddot{w}_{0}}{\partial y} \\
& +c_{1}\left(-I_{4} \ddot{\phi}_{2}-c_{1} I_{6} \ddot{\phi}_{2}+c_{1} I_{6} \frac{\partial \ddot{w}_{0}}{\partial y}\right)
\end{aligned}
$$$$
+c_{40} \frac{\partial^{2} v_{0}}{\partial x \partial y} \frac{\partial w_{0}}{\partial x}-c_{41} \frac{\partial u_{0}}{\partial y} \frac{\partial w_{0}}{\partial x}+c_{42} \frac{\partial^{2} u_{0}}{\partial y^{2}} \frac{\partial w_{0}}{\partial x}
$$$$
-c_{43} \frac{\partial v_{0}}{\partial y} \frac{\partial w_{0}}{\partial x}+c_{44} \frac{\partial u_{0}}{\partial x} \frac{\partial^{2} w_{0}}{\partial y^{2}}+c_{45} v_{0} \frac{\partial^{2} w_{0}}{\partial y^{2}}
$$$$
+c_{46} \frac{\partial v_{0}}{\partial y} \frac{\partial w_{0}}{\partial y}+c_{47} w_{0} \frac{\partial^{2} w_{0}}{\partial y^{2}}+c_{48} \frac{\partial v_{0}}{\partial y} \frac{\partial^{2} w_{0}}{\partial y^{2}}
$$$$
+c_{49} \frac{\partial^{2} v_{0}}{\partial^{2} y} \frac{\partial w_{0}}{\partial y}+c_{50} u_{0} \frac{\partial^{2} w_{0}}{\partial y^{2}}+c_{51} \frac{\partial u_{0}}{\partial y} \frac{\partial w_{0}}{\partial y}
$$$$
+c_{52} \frac{\partial^{3} \phi_{1}}{\partial x^{3}}+c_{53} \frac{\partial^{3} \phi_{2}}{\partial y^{3}}+c_{54} \frac{\partial^{3} \phi_{1}}{\partial x^{2} \partial y}+c_{55} \frac{\partial^{3} \phi_{2}}{\partial x \partial y^{2}}
$$

where $c_{1}=4 / 3 h^{2}, c_{2}=3 c_{1}$, and $\mu$ in (6c) is the damping coefficient, and all the parameters of the specific expression can be found in the Appendix.

The boundary conditions of the cantilever shell are expressed as

$$
\begin{aligned}
x & =0: N_{x y}=M_{x x}=M_{x y}-c_{1} P_{x y}=\bar{Q}_{x} \\
& =0, \\
x & =a: N_{x y}=M_{x x}=M_{x y}-c_{1} P_{x y}=\bar{Q}_{x} \\
& =0, \\
y & =0: u_{0}=v_{0}=w_{0}=\phi_{1}=\phi_{2}=0,
\end{aligned}
$$




$$
\begin{aligned}
y & =b: N_{y y}=N_{x y}=M_{y y}=M_{x y}-c_{1} P_{x y} \\
& =\bar{Q}_{y}=0, \\
\int_{-h / 2}^{h / 2} N_{x x} d z & = \pm \int_{-h / 2}^{h / 2} q d z, \quad(x=0, a) .
\end{aligned}
$$

\section{Perturbation Analysis}

Since vibration amplitudes of the lower frequencies are much larger than that of the higher frequencies for the shell, the mainly dynamical damage or instability of structures is caused by resonances in lower frequencies. Here, the first two modes of the MFC laminated shell are considered. Thus, displacements $u_{0}, v_{0}, w_{0}, \varphi_{1}$, and $\varphi_{2}$, which satisfy the boundary conditions for the shell, are represented as

$$
\begin{aligned}
u_{0}= & u_{1} \sin \frac{\pi x}{2} \cos \pi y+u_{2} \sin \frac{3 \pi x}{2} \cos 2 \pi y, \\
v_{0}= & v_{1} \sin \frac{\pi x}{2} \sin \pi y+v_{2} \sin \frac{3 \pi x}{2} \sin 2 \pi y, \\
\phi_{1}= & \phi_{11} \sin \frac{\pi x}{2} \cos \pi y+\phi_{12} \sin \pi x \cos 2 \pi y, \\
\varphi_{2}= & \varphi_{21}\left(1-\cos \frac{\pi x}{2}\right) \sin \pi y \\
& +\varphi_{22}\left(1-\cos \frac{\pi x}{2}\right) \sin 2 \pi y, \\
w_{0}= & w_{1}(t) X_{1}(x) Y_{1}(y)+w_{2}(t) X_{2}(x) Y_{2}(y),
\end{aligned}
$$

where

$$
\begin{aligned}
X_{i}(x)= & \sin \lambda_{i} x-\sinh \lambda_{i} x \\
& +\alpha_{i}\left(\cosh \lambda_{i} x-\cos \lambda_{i} x\right), \\
Y_{1}(y)= & 1, \\
Y_{2}(y)= & \sqrt{3}\left(1-\frac{2 y}{b}\right),
\end{aligned}
$$

To obtain the dimensionless equations, the transformation of variables and parameters are introduced as

$$
\begin{aligned}
& \bar{u}_{0}=\frac{u_{0}}{\sqrt{R h}}, \\
& \bar{v}_{0}=\frac{v_{0}}{\sqrt{R h}}, \\
& \bar{w}_{0}=\frac{w_{0}}{R}, \\
& \bar{\varphi}_{1}=\varphi_{1},
\end{aligned}
$$

$$
\begin{aligned}
& \bar{\varphi}_{2}=\varphi_{2}, \\
& \bar{x}=\frac{x}{\sqrt{R h}}, \\
& \bar{y}=\frac{y}{\sqrt{R h}}, \\
& \bar{z}=\frac{z}{h}, \\
& \bar{R}_{1}=\frac{R_{1}}{R}, \\
& \bar{R}_{2}=\frac{R_{2}}{R}, \\
& \bar{I}_{i}=\frac{1}{L^{i+1} \rho} I_{i}, \\
& \bar{F}=\frac{(R h)^{7 / 2}}{E h^{7}} F, \\
& \bar{P}=\frac{b^{2}}{E l^{3}} P, \\
& \bar{\mu}=\frac{1}{\sqrt{\rho E}} \frac{a^{2} b^{2}}{h^{2}} \mu, \\
& \bar{T}=\frac{1}{L} \sqrt{\frac{E}{\rho}} T, \\
& {[\bar{A}]=\frac{1}{E L}[A] \text {, }} \\
& {[\bar{B}]=\frac{1}{E L^{2}}[B],} \\
& {[\bar{D}]=\frac{1}{E L^{3}}[D],} \\
& {[\bar{E}]=\frac{1}{E L^{4}}[E],} \\
& {[\bar{F}]=\frac{1}{E L^{5}}[F],} \\
& {[\bar{H}]=\frac{1}{E L^{7}} H \text {, }} \\
& L=\sqrt{R h} .
\end{aligned}
$$

Then, taking all these derived expressions in (8a), (8b), (8c), (8d), and (8e)-(9a), (9b), (9c), (9d), (9e), and (9f) into (6a), (6b), (6c), (6d), and (6e) and applying the Galerkin procedure, a two-degree-of-freedom nonlinear ordinary differential equation of the MFC laminated shell with dimensionless is obtained as follows:

$$
\begin{aligned}
& \ddot{w}_{1}+\mu \dot{w}_{1}+\omega_{1}^{2} w_{1}+\alpha_{11} w_{1}^{2}+\alpha_{12} w_{2}^{2}+\alpha_{13} w_{1} w_{2} \\
& +\alpha_{14} w_{1} \cos \Omega_{2} t+\alpha_{15} w_{2} \cos \Omega_{2} t=\alpha_{16} f \cos \Omega_{1} t
\end{aligned}
$$




$$
\begin{aligned}
\ddot{w}_{2} & +\mu \dot{w}_{2}+\omega_{2}^{2} w_{2}+\alpha_{21} w_{1}^{2}+\alpha_{22} w_{2}^{2}+\alpha_{23} w_{1} w_{2} \\
& +\alpha_{24} w_{1} \cos \Omega_{2} t+\alpha_{25} w_{2} \cos \Omega_{2} t=\alpha_{26} f \cos \Omega_{1} t
\end{aligned}
$$

Here, $\alpha_{14}, \alpha_{15}, \alpha_{24}$, and $\alpha_{25}$ present the piezoelectric coefficients of the shell.

Considering the case of primary parametric and 1:1 internal resonance, the following relations can be established accordingly:

$$
\begin{aligned}
& \omega_{1}^{2}=\Omega_{1}^{2}+\varepsilon \sigma_{1}, \\
& \omega_{2}^{2}=\Omega_{1}^{2}+\varepsilon \sigma_{2}, \\
& \omega_{1}^{2} \approx \omega_{2}^{2}, \\
& \Omega_{1}=\Omega_{2}=1,
\end{aligned}
$$

where $\sigma_{1}$ and $\sigma_{2}$ are the detuning parameters.

To study the oscillations and bifurcations of the established nonlinear system, the method of multiscale is a powerful tool to determine the solutions for conservative and nonconservative systems. Hence, this method is adopted to investigate the nonlinear vibration responses of the MFC shell.

The uniformly approximate solutions of (11a) and (11b) are obtained as

$$
\begin{aligned}
& w_{1}=w_{10}\left(T_{0}, T_{1}\right)+\varepsilon w_{11}\left(T_{0}, T_{1}\right)+\cdots, \\
& w_{2}=w_{20}\left(T_{0}, T_{1}\right)+\varepsilon w_{21}\left(T_{0}, T_{1}\right)+\cdots,
\end{aligned}
$$

where $T_{0}=t$ and $T_{1}=\varepsilon t$.

Then, the derivatives with respect to $t$ become

$$
\begin{aligned}
\frac{d}{d t} & =D_{0}+\varepsilon D_{1}, \\
\frac{d^{2}}{d t^{2}} & =D_{0}^{2}+2 \varepsilon D_{0} D_{1} .
\end{aligned}
$$

Substituting (13a), (13b), (14a), and (14b) into (11a) and (11b) and equating the coefficients of $\varepsilon$ to zero yield the following differential equations:

$$
\begin{aligned}
& \varepsilon^{0} \\
& D_{0} w_{10}+\Omega_{1}^{2} w_{10}=0 \text {, } \\
& D_{0} w_{20}+\Omega_{1}^{2} w_{20}=0 \text {, } \\
& \varepsilon^{1} \\
& D_{0} w_{11}+\Omega_{1}^{2} w_{11}=-2 D_{0} D_{1} w_{10}-\mu D_{0} w_{10}-\sigma_{1} w_{10} \\
& -\alpha_{11} w_{10}^{2}-\alpha_{12} w_{20}^{2} \\
& -\alpha_{13} w_{10} w_{20}-\alpha_{14} w_{10} \cos \Omega_{2} t \\
& -\alpha_{15} w_{20} \cos \Omega_{2} t \\
& +\alpha_{16} f \cos \Omega_{1} t \text {, }
\end{aligned}
$$

$$
\begin{aligned}
D_{0} w_{21}+\Omega_{1}^{2} w_{21}= & -2 D_{0} D_{1} w_{20}-\mu D_{0} w_{20}-\sigma_{1} w_{20} \\
& -\alpha_{21} w_{10}^{2}-\alpha_{22} w_{20}^{2} \\
& -\alpha_{23} w_{10} w_{20}-\alpha_{24} w_{10} \cos \Omega_{2} t \\
& -\alpha_{25} w_{20} \cos \Omega_{2} t \\
& +\alpha_{26} f \cos \Omega_{1} t
\end{aligned}
$$

The averaged equations in the Polar Coordinates form are obtained for the MFC laminated shell as follows:

$$
\begin{aligned}
\dot{a}_{1} & =-\frac{1}{2} \mu a_{1}+\frac{1}{2} \alpha_{16} f \sin \theta_{1}, \\
a_{1} \dot{\theta}_{1} & =\frac{1}{2} \sigma_{1} a_{1}+\frac{1}{4} \alpha_{14} a_{1}+\frac{1}{4} \alpha_{15} a_{2}-\frac{1}{2} \alpha_{16} f \cos \theta_{1}, \\
\dot{a}_{2} & =-\frac{1}{2} \mu a_{2}+\frac{1}{2} \alpha_{26} f \sin \theta_{2}, \\
a_{2} \dot{\theta}_{2} & =\frac{1}{2} \sigma_{2} a_{2}+\frac{1}{4} \alpha_{24} a_{1}+\frac{1}{4} \alpha_{25} a_{2}-\frac{1}{2} \alpha_{26} f \cos \theta_{2} .
\end{aligned}
$$

\section{Numerical Simulation}

In this section, a series of numerical experiments are conducted for the nonlinear dynamical behavior of the MFC laminated shell. The nonlinear governing equations (17a), (17b), (17c), and (17d) are used to perform the numerical simulation through the Runge-Kutta algorithm. Whereas the transverse excitation is one of the most important factors on the nonlinear vibration of the system, the excitation $f_{1}$ is chosen as a controlling parameter in the subsequent studies.

Choose the following parameters: $\mu=0.6, \alpha_{11}=29.2$, $\alpha_{12}=-6.7, \alpha_{13}=10.7, \alpha_{15}=1.0, \alpha_{16}=25.3, \alpha_{21}=27.7, \alpha_{22}=$ $5.2, \alpha_{23}=27.0, \alpha_{24}=1.0, \alpha_{25}=1.0, \alpha_{26}=6.9, x_{1}=1.6, x_{2}=$ $0.3, x_{3}=1.9$, and $x_{1}=0.9$. The frequency-response curves of the first-order and second-order are obtained through changing the initial condition of the MFC shell. The behavior shown in Figure 3 features the characteristics of soften-type systems with the piezoelectric coefficient $\alpha_{14}$ increased from 0.5 to 1.5 . It is also shown that the stiffness would be decreased with the increase in $\alpha_{14}$.

The bifurcation diagrams of Poincare sections for the displacements of the middle surface of the shell are shown in Figure 4 with the transverse excitation increasing from 700 to 2000 . The complex nonlinear vibration responses of the system are discussed corresponding to the above parameters. The system keeps the chaotic motion until $f_{1}$ increased to 800 , at which point period motions of the system occur. After short windows of period- $n$ motions occur in 950-1000, the motions of the system dominantly enter into chaotic motions thereafter. Then the chaotic motion is observed again until the transverse excitation $f_{1}$ equals 1500 . The motion status of the MFC laminated shell alternately appears from unstable to stable and then again to unstable, which depends on the amplitude of the transverse excitation $f_{1}$.

To reveal the specific form of different sections in the bifurcation diagram, the response-frequency curves, phase 


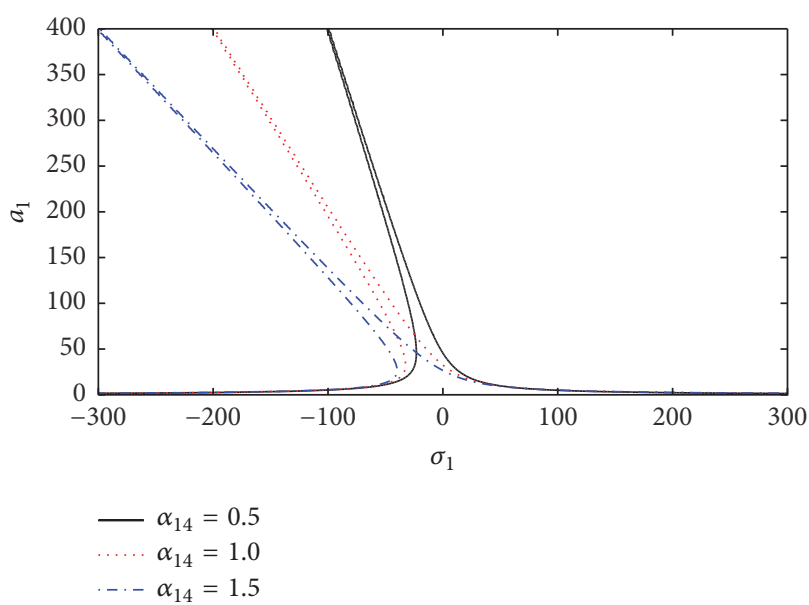

(a)

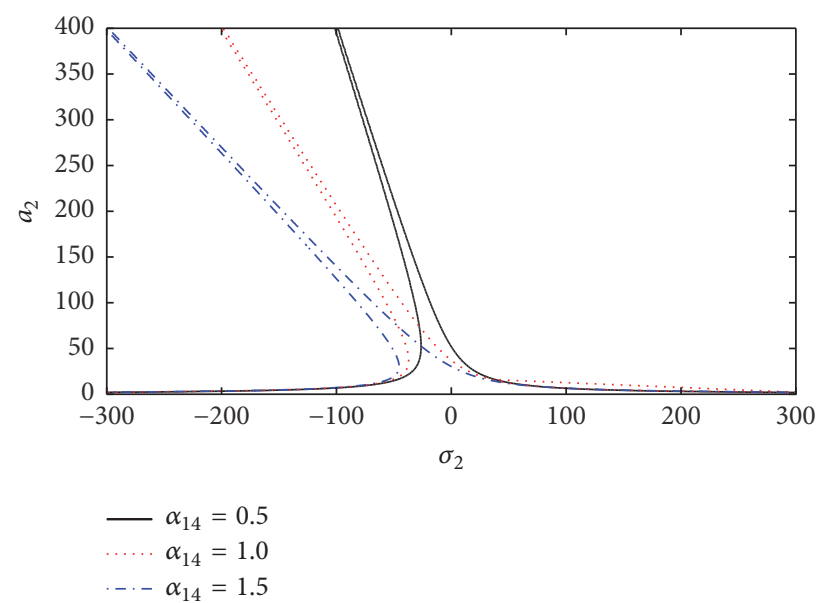

(b)

FIGURE 3: Response-frequency curves.

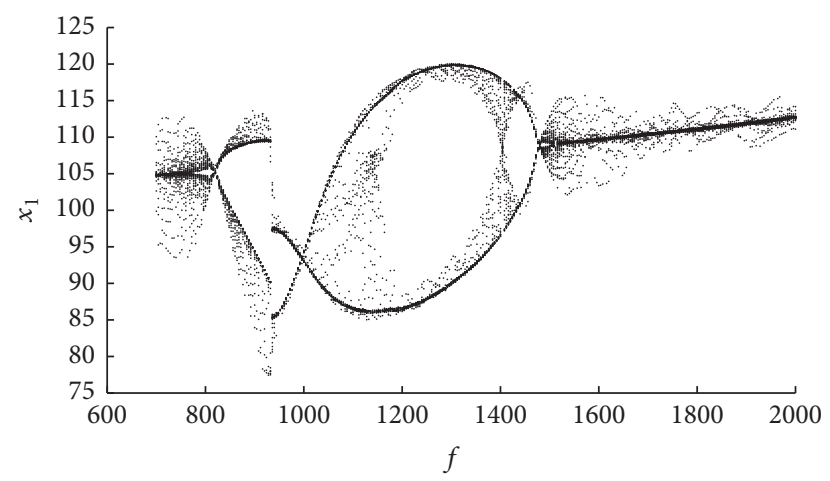

(a)

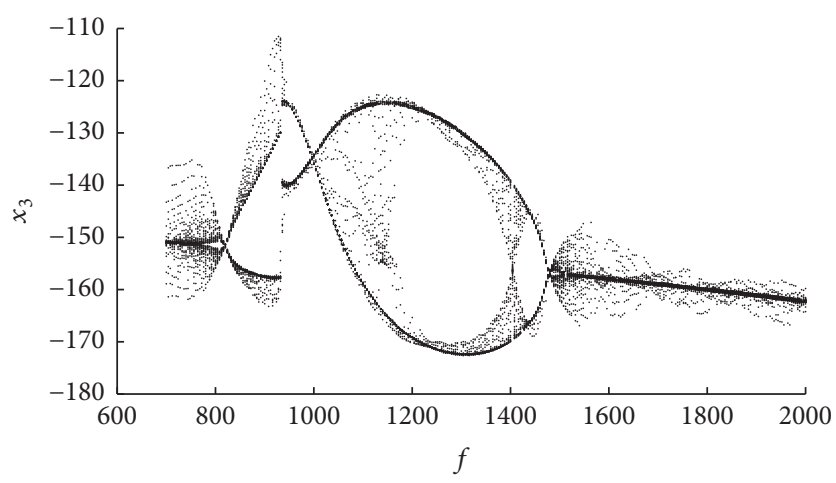

(b)

FIgURE 4: The bifurcation diagram.

portraits, power spectrums, and waveforms of the shell are depicted as shown in Figures 5(a)-5(f). Diagrams (a) and (c) are, respectively, the wave forms on the planes $\left(t, x_{1}\right)$ and $\left(t, x_{3}\right)$, and Diagrams (b) and (d) are the two-dimensional phase portraits on the planes $\left(x_{1}, x_{2}\right)$ and $\left(x_{3}, x_{4}\right)$. Diagrams (e) show the three-dimensional phase portraits in the space $\left(x_{1}, x_{2}, x_{3}\right)$ while Diagrams (f) represent the Poincare diagram. The typical chaotic motion of the system is given as in Figure 5 when the amplitude of the transverse excitation arrives at 760 .

From the frequency-response curves of the system analyzed, it is known that the structure stiffness would alter when the piezoelectric coefficients of the system changed, so it would be effective to adjust the motion status of the MFC shell from unstable to stable through modulating the piezoelectric coefficients of the system. Fix the system parameters, based on which Figure 5 is depicted, and only adjust the piezoelectric parameters $\alpha_{25}$ to perform numerical simulations and the results are shown in Figure 6. The bifurcation diagram reveals that the system would enter into periodic motions when $\alpha_{25}$ is increased from 0 to 1.6. The specific periodic motion form of the system is also shown in Figure 7 when $\alpha_{25}$ is equal to 2 .

In order to describe the influence of the piezoelectric parameters on the nonlinear vibrations of the system profoundly, another set of parameters is selected in the following: $\mu=0.6, \alpha_{11}=-5.3, \alpha_{12}=8.9, \alpha_{13}=26.4, \alpha_{14}=1.0, \alpha_{15}=$ $1.0, \alpha_{16}=19.3, \alpha_{21}=-10.4, \alpha_{22}=7.4, \alpha_{23}=-22.4, \alpha_{24}=1.0$, $\alpha_{25}=1.0, \alpha_{26}=14.1, x_{1}=2.6, x_{2}=1.3, x_{3}=4.5$, and $x_{1}=5.1$. It is found from the bifurcation diagram in Figure 8 that the response of the system varies from periodic motions to chaotic motions when the transversal excitation increases from 3 to 18 . The different periodic and chaotic motions of the system are also given as in Figures 9-10, respectively, when $f_{1}$ equals 5 and 12 .

Based on the motion of the system in Figure 8 , we only increase the piezoelectric parameter $\alpha_{25}$ to describe the vibration responses of the system. The bifurcation diagram of the system also shows the evolution from chaos to period$n$ and then back into chaos again as shown in Figure 11. It is also found that the system enters into periodic motion from chaotic motion when the piezoelectric parameter $\alpha_{25}$ 


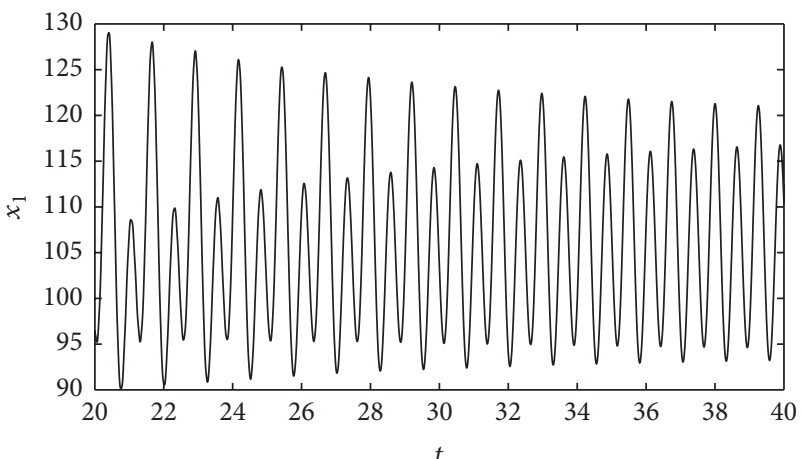

(a)

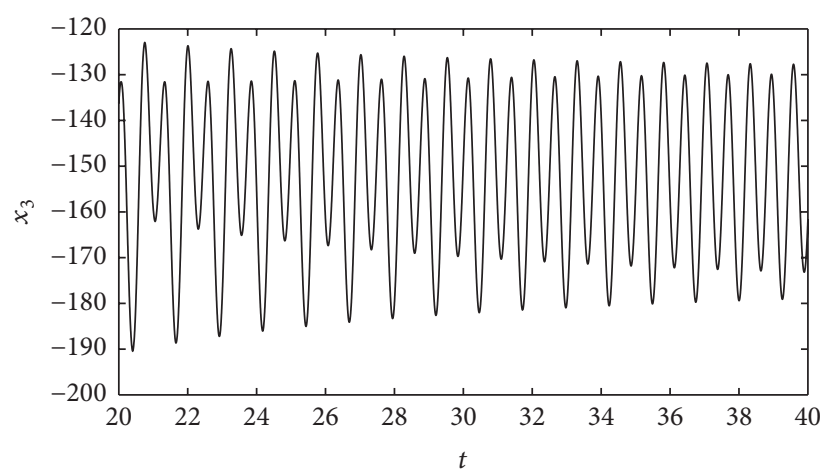

(c)

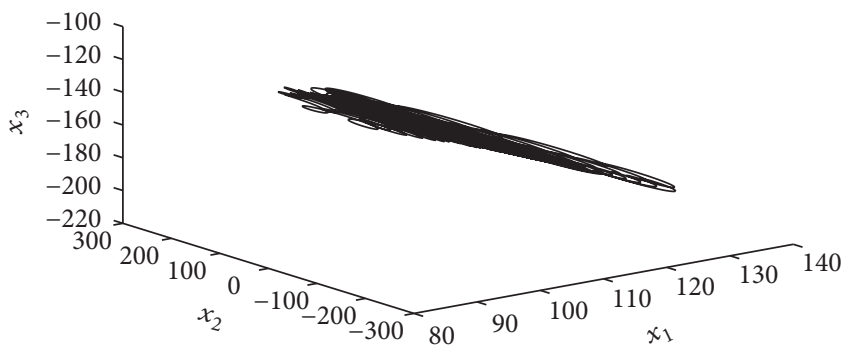

(e)

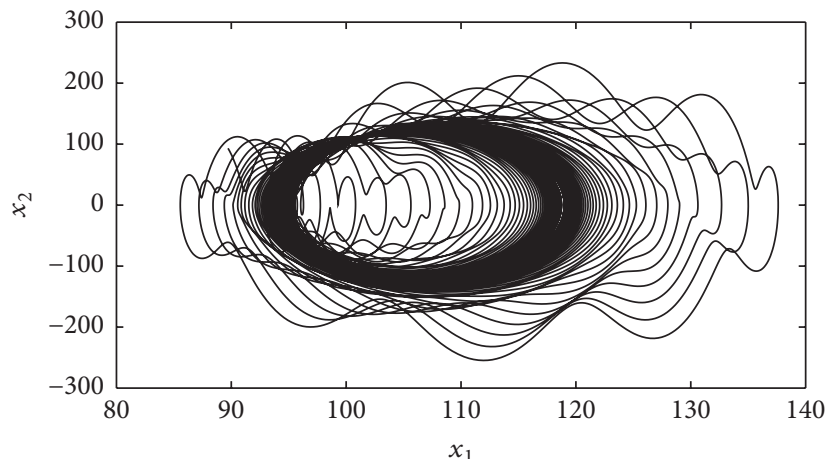

(b)

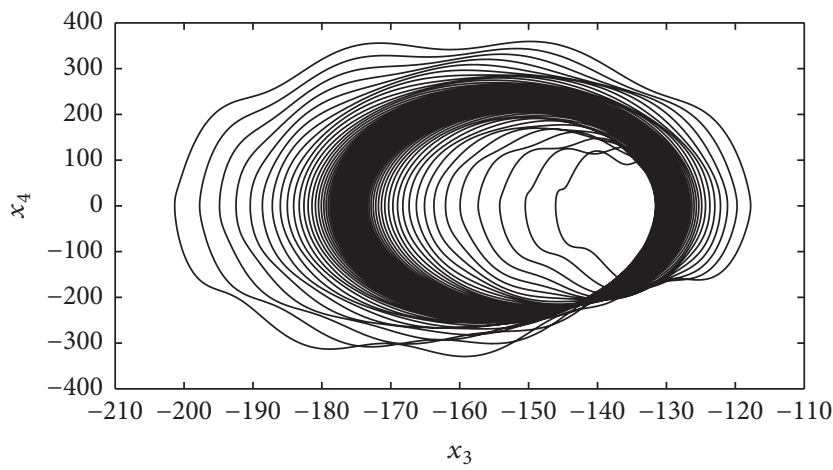

(d)

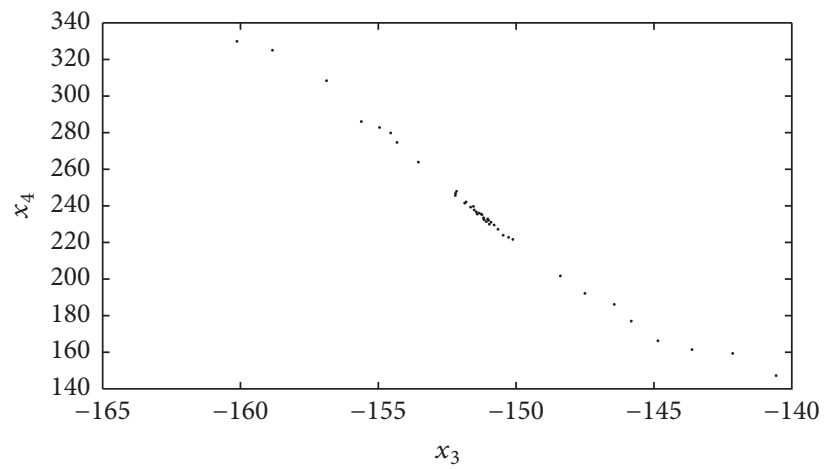

(f)

FIGURE 5: The chaotic motion of the shell.

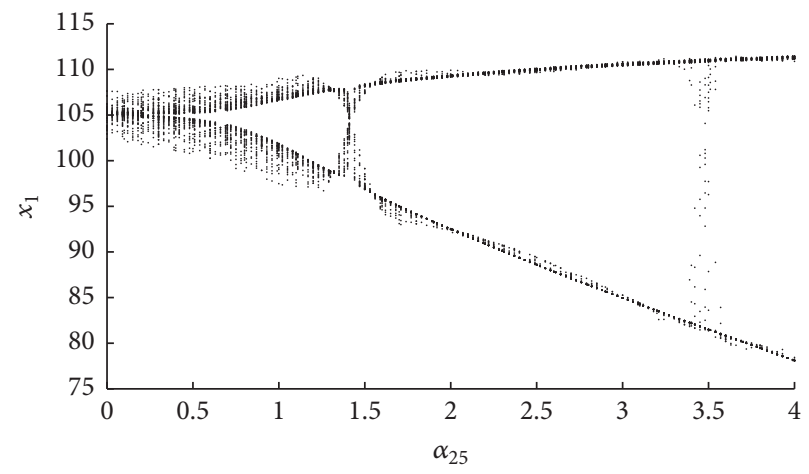

(a)

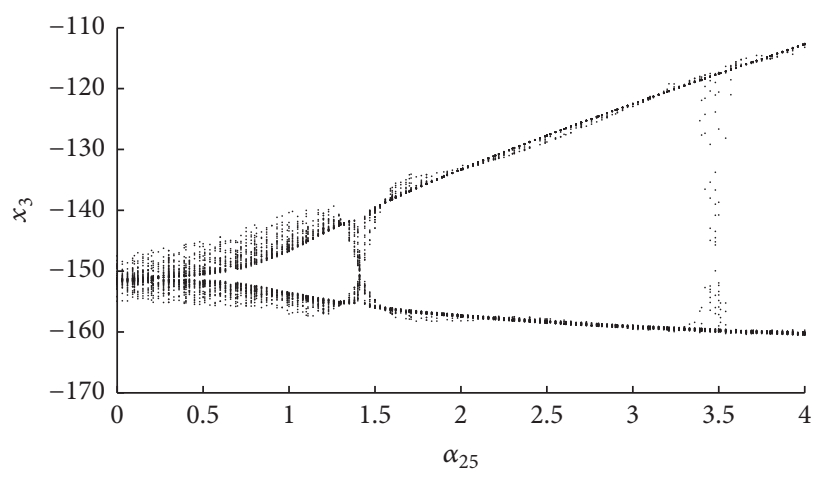

(b)

Figure 6: The bifurcation diagram of the shell. 


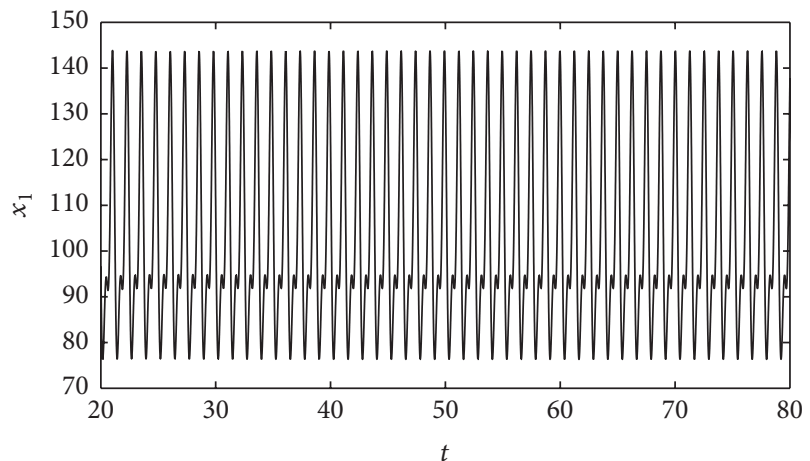

(a)

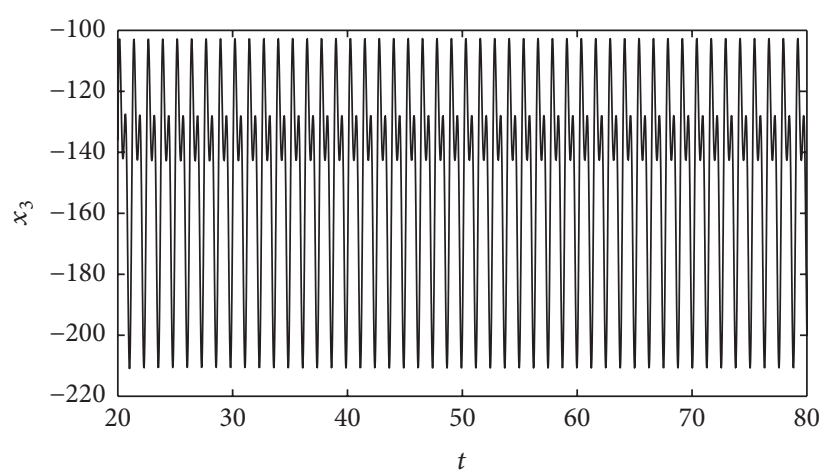

(c)

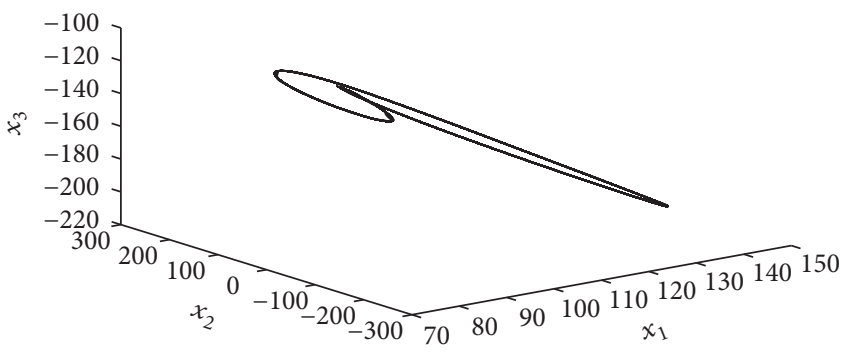

(e)

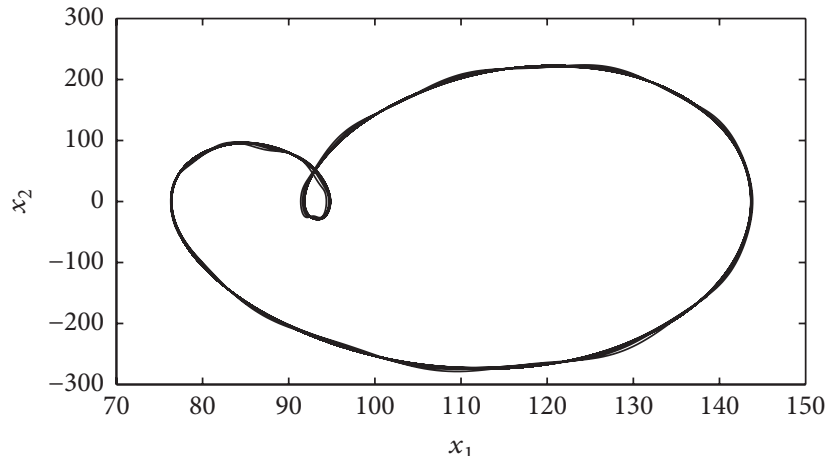

(b)

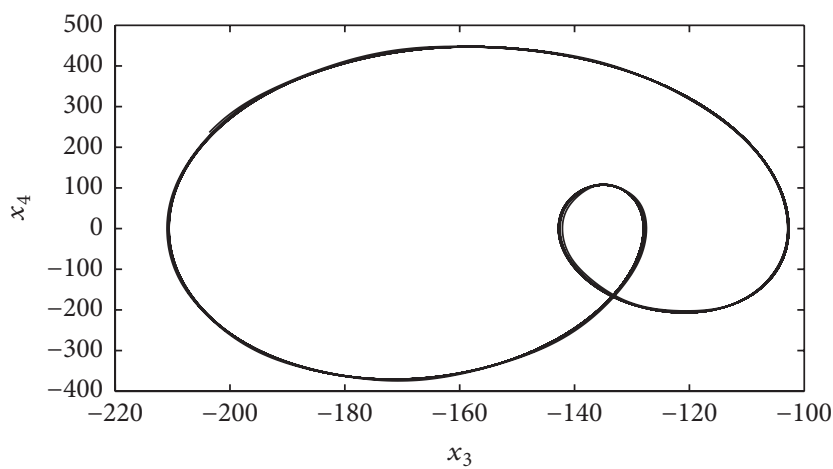

(d)

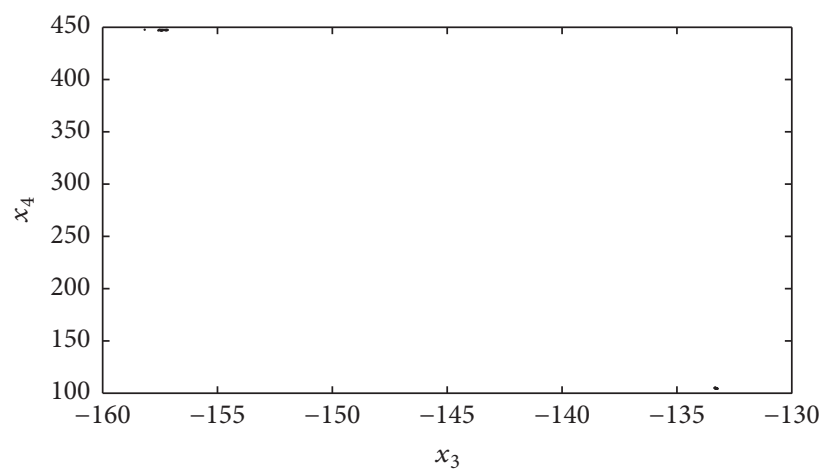

(f)

FIgURE 7: The periodic motion of the shell.

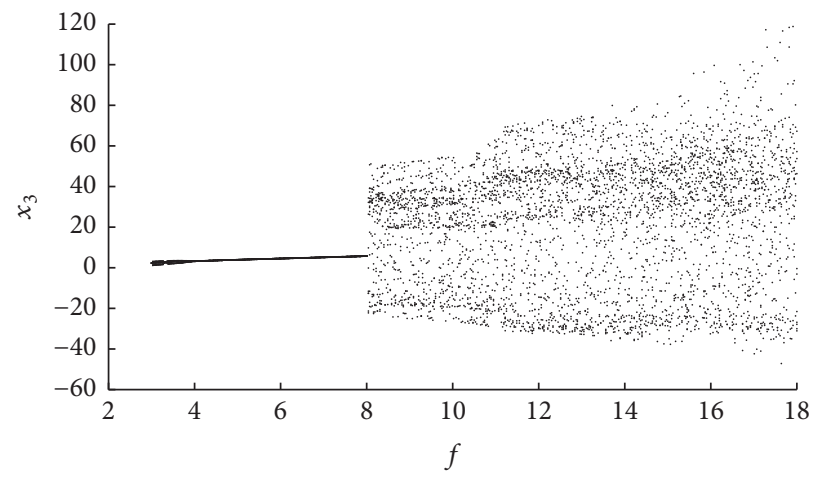

(a)

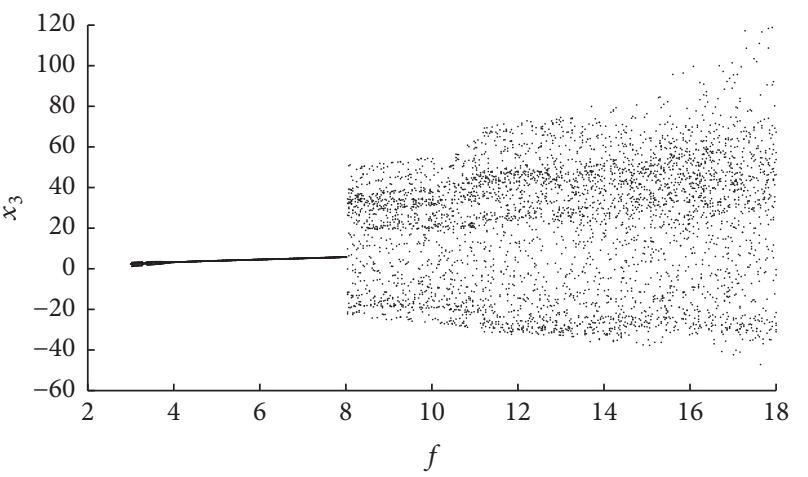

(b)

FIGURE 8: The bifurcation diagram of the shell. 


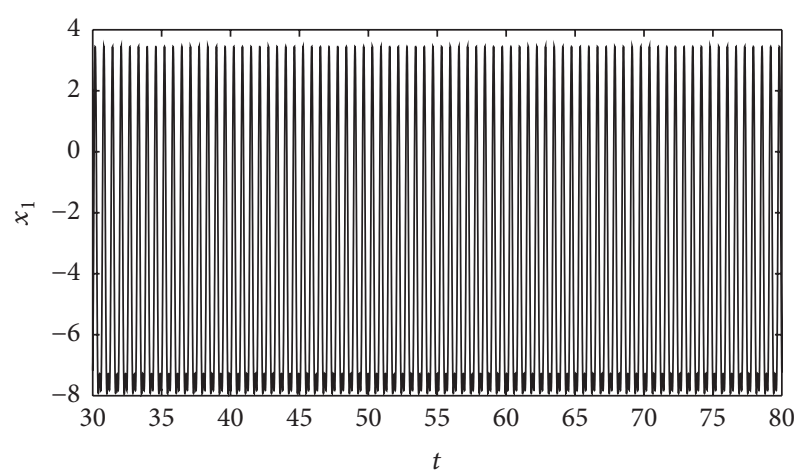

(a)

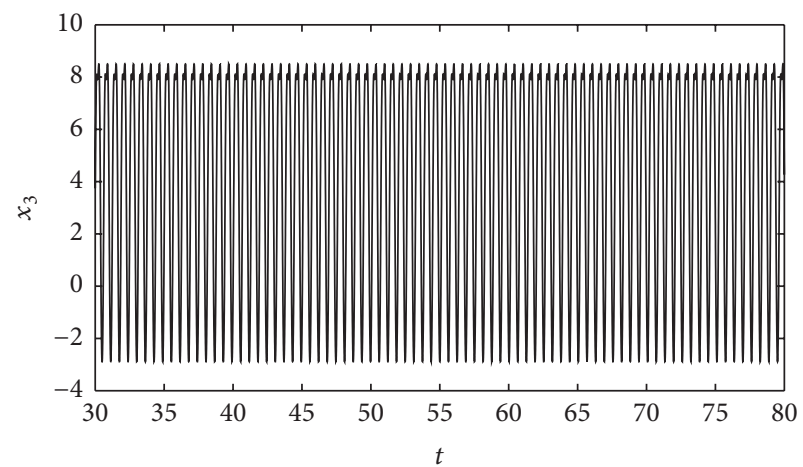

(c)

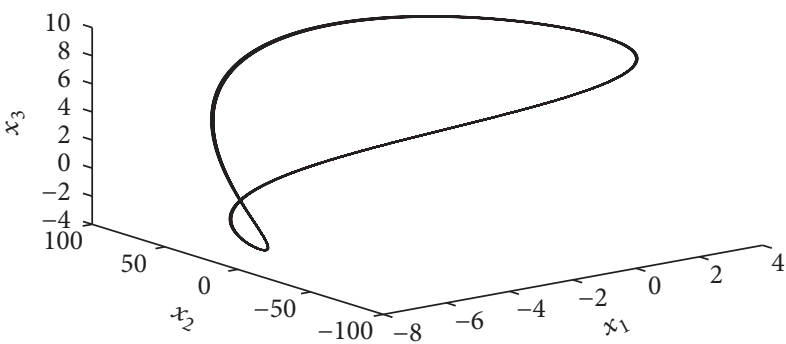

(e)

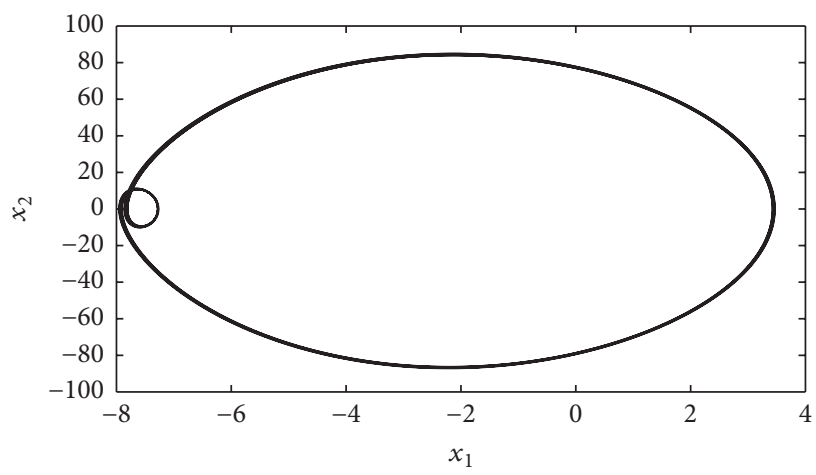

(b)

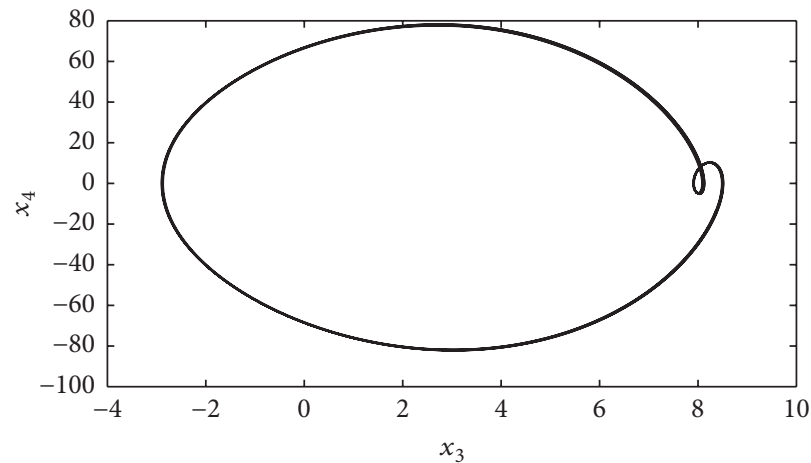

(d)

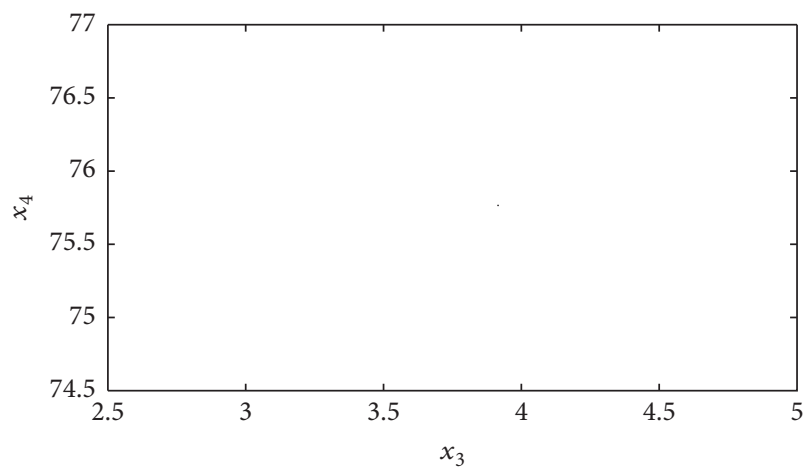

(f)

FIGURE 9: The periodic motion of the shell.

increases from 1.0 to 2.0. Figure 12 shows the specific periodic motion of the system as $\alpha_{25}$ equals 1.5 .

From the results of the numerical simulation, it is discovered that the piezoelectric parameters could adjust the vibration responses of the structure effectively. Since the piezoelectric parameters could vary the stiffness of the shell, it is generalized: once the inherent frequency of the system changes, the resonance would be restrained for piezoelectric structures.

\section{Conclusion}

In this paper, nonlinear dynamical behaviors of a simply supported cantilever MFC shell are presented, which is subjected to transverse loads. Based on known geometrical and material properties of its constituents, their electric field dependence is presented. The vibration mode-shape functions are obtained according to the boundary conditions, and then Galerkin method is employed to transform the partial differential equations into two nonlinear ordinary differential equations. The illustrative case of $1: 1$ internal resonances is considered. The externally excited system is transformed into a set of averaged equations by using the method of multiple scales. Next, the effect of the transverse excitations and the piezoelectric coefficients on the MFC laminated shell is described in numerical simulation.

The results of the numerical simulation demonstrate the complex nonlinear vibration responses of the MFC shell that occurred under the transverse excitation, including the periodic and chaotic motions. The energy transformation existed 


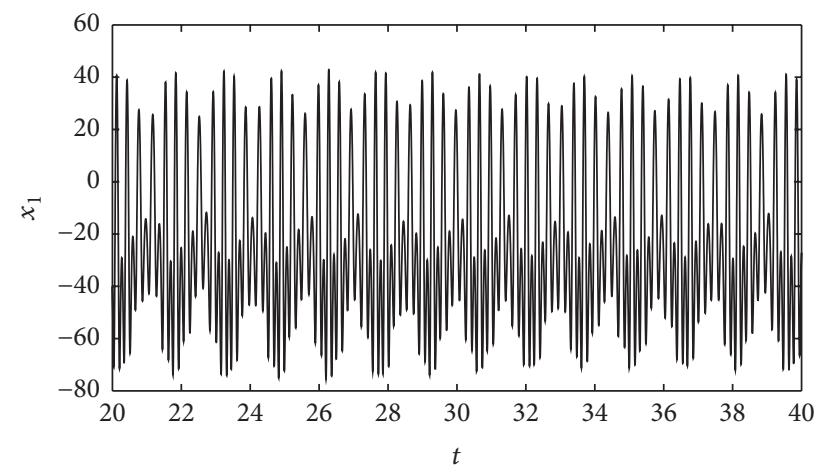

(a)

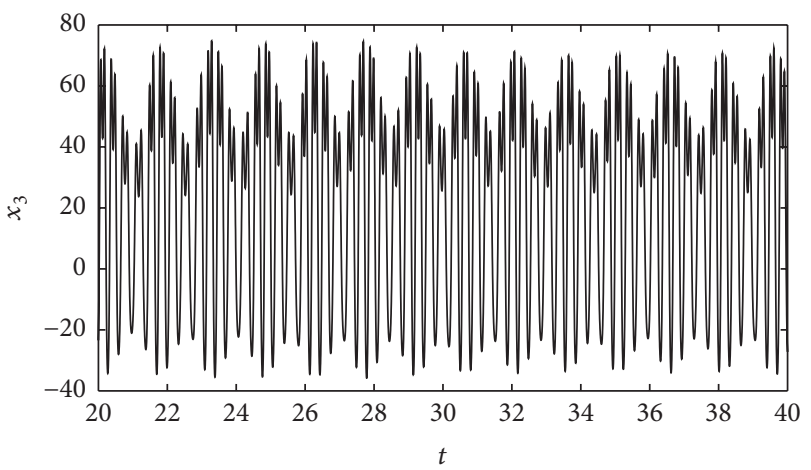

(c)

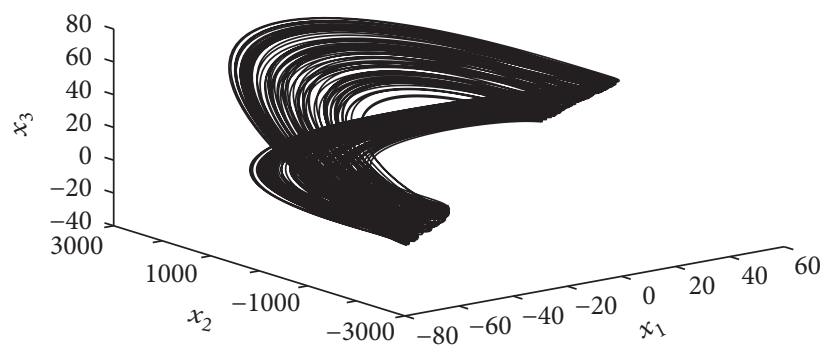

(e)

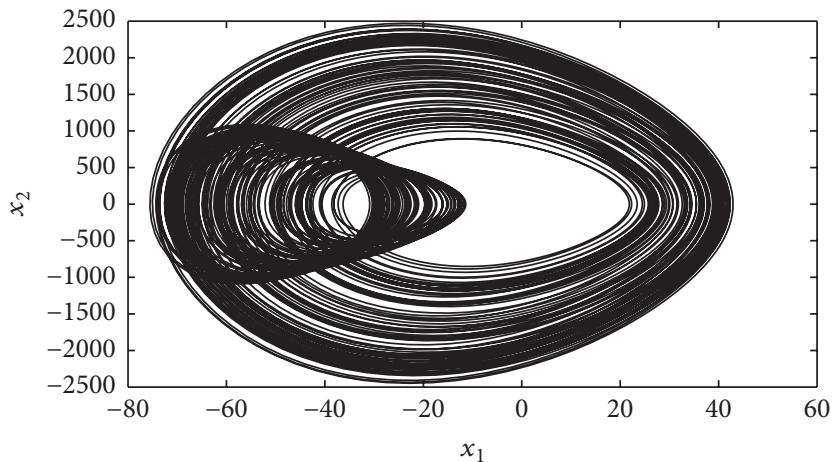

(b)

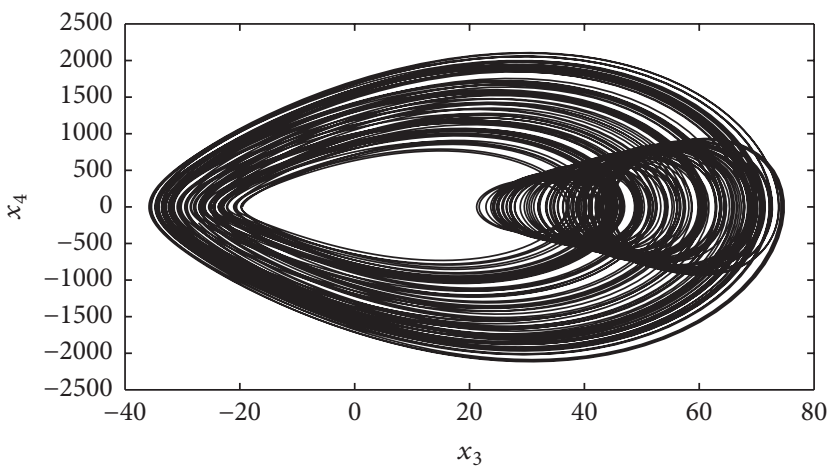

(d)

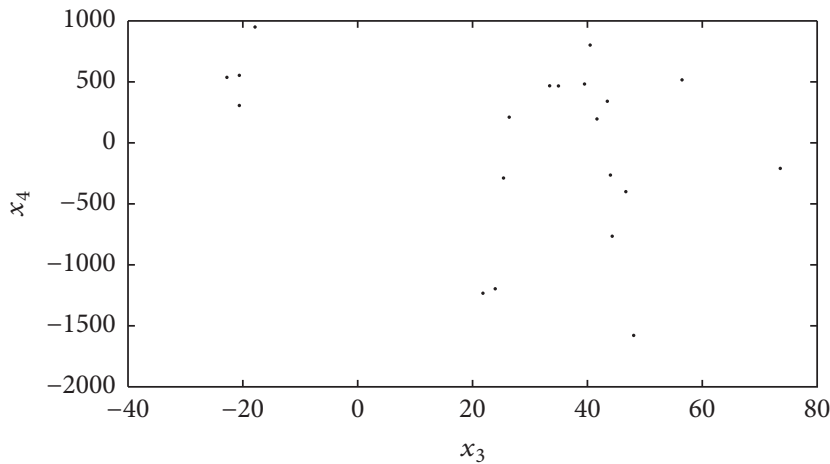

(f)

FIGURE 10: The chaotic motion of the shell.

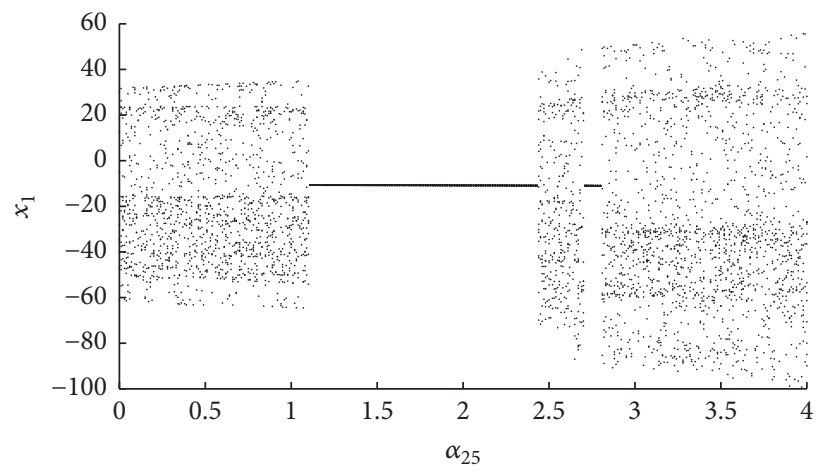

(a)

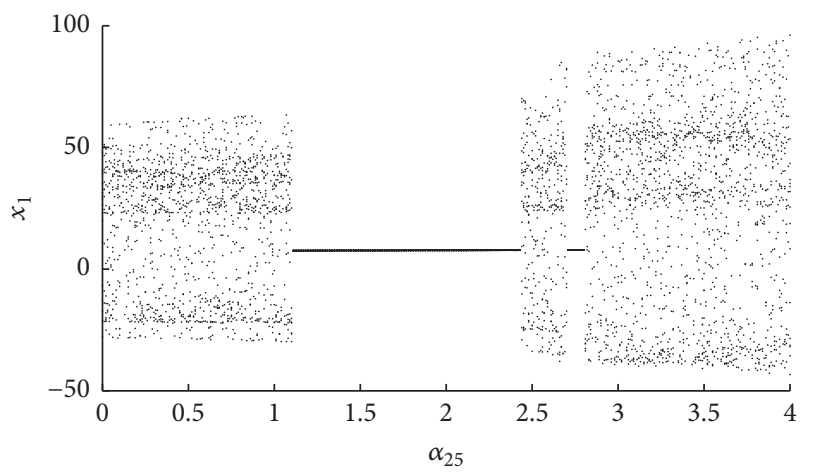

(b)

FIGURE 11: The bifurcation diagram of the shell. 


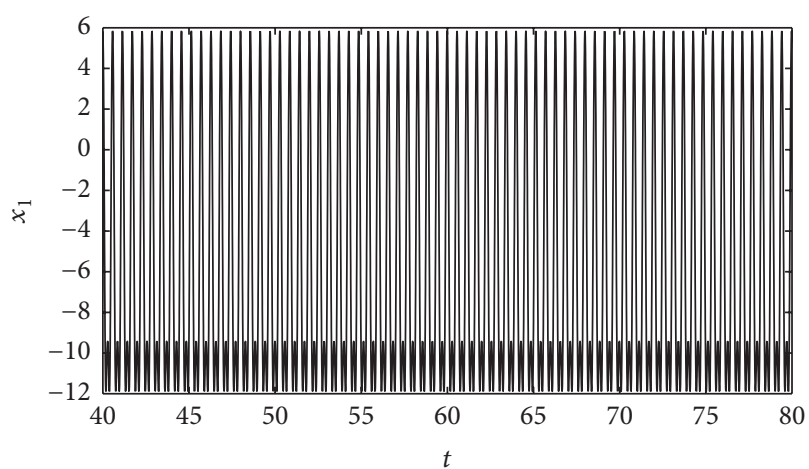

(a)

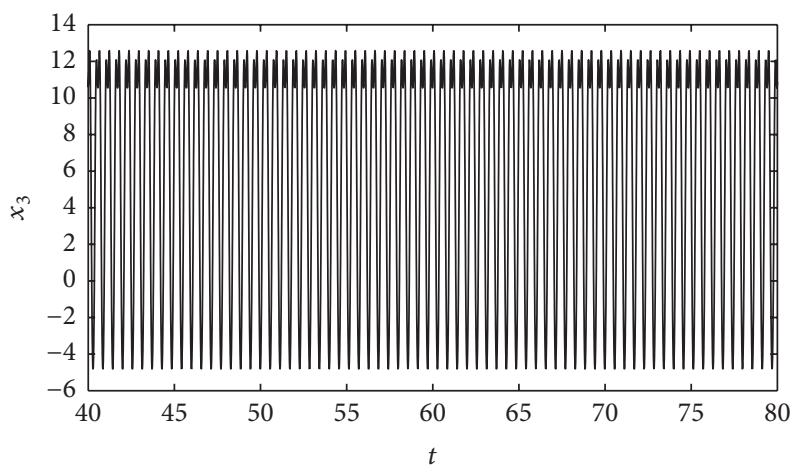

(c)

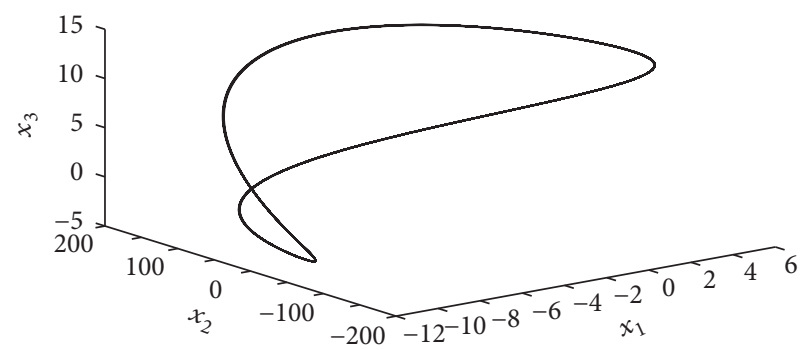

(e)

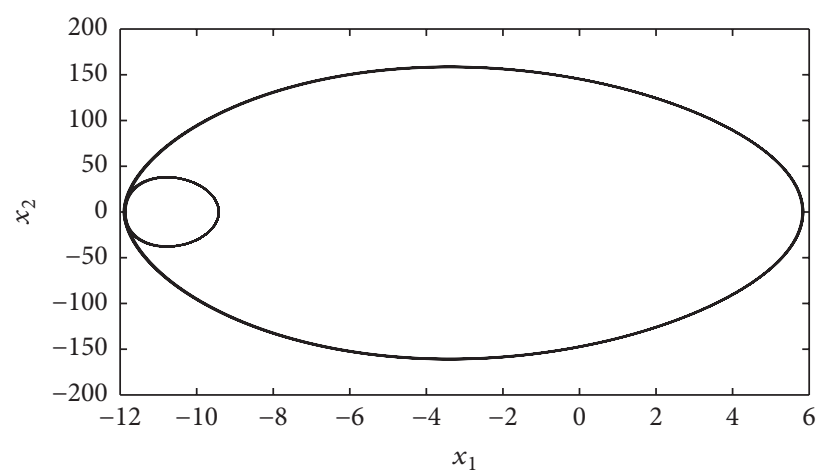

(b)

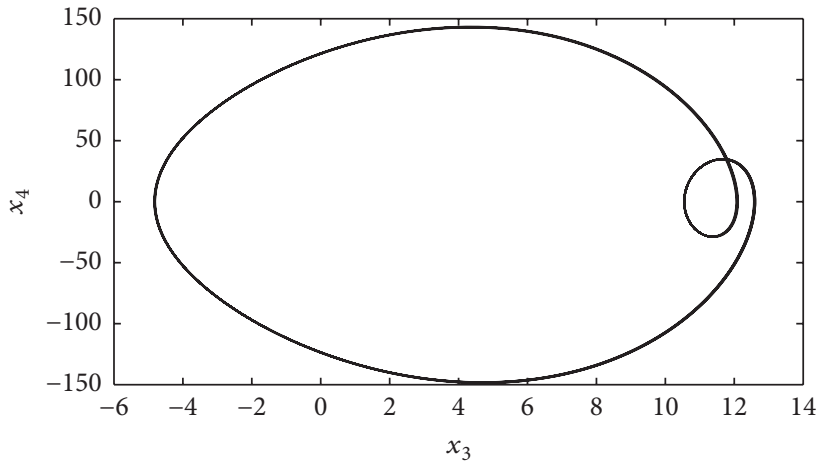

(d)

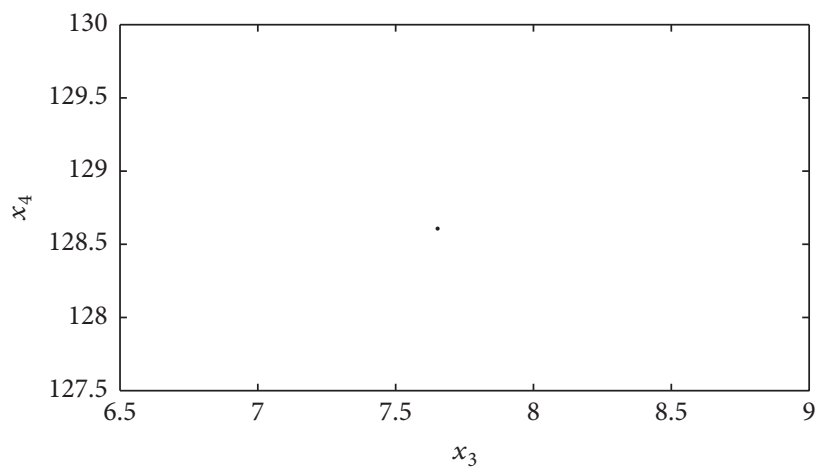

(f)

FIgURE 12: The periodic motion of the shell.

between two resonances modes. The appropriate control technique of the forcing excitations contributes significantly to the responses of autonomous nonlinear systems. It is also revealed that the piezoelectric parameters of the structure could adjust the dynamic stability of the structure from unstable to stable which would be a good way to control vibration responses for MFC structures.

\section{Appendix}

$$
\begin{aligned}
& a_{11}=\frac{1}{a_{1}} A_{11}, \\
& a_{12}=\frac{1}{a_{2}} A_{66},
\end{aligned}
$$

$$
\begin{aligned}
& a_{13}=\left(\frac{1}{a_{2}} A_{12}+\frac{1}{a_{1}} A_{66}\right), \\
& a_{14}=\frac{1}{a_{1} a_{2}} \frac{\partial a_{2}}{\partial x} A_{12}, \\
& a_{15}=\frac{1}{a_{1} a_{2}} \frac{\partial a_{1}}{\partial y} A_{11}, \\
& a_{16}=\frac{1}{a_{1} a_{2}} \frac{\partial a_{1}}{\partial y} A_{66} \\
& a_{17}=\frac{1}{a_{1} a_{2}} \frac{\partial a_{2}}{\partial x} A_{66}, \\
& a_{18}=\frac{c_{1}^{2}}{R_{1} a_{1}} H_{11},
\end{aligned}
$$




$$
\begin{aligned}
& a_{19}=\frac{c_{1}^{2}}{R_{1}}\left(\frac{1}{a_{2}} H_{12}+\frac{1}{a_{1}} H_{66}+\frac{1}{a_{2}} H_{66}\right), \quad b_{17}=\frac{1}{a_{1} a_{2}} \frac{\partial a_{1}}{\partial y} A_{66}, \\
& a_{20}=\frac{c_{1}^{2}}{R_{1} a_{1} a_{2}} \frac{\partial a_{2}}{\partial x} H_{12} \\
& b_{18}=\frac{c_{1}^{2}}{R_{2} a_{2}} H_{22} \\
& a_{21}=\frac{c_{1}^{2}}{R_{1} a_{1} a_{2}} \frac{\partial a_{2}}{\partial x} H_{66} \\
& b_{19}=\frac{c_{1}^{2}}{R_{1}}\left(\frac{1}{a_{1}} H_{21}+\frac{1}{a_{2}} H_{66}+\frac{1}{a_{1}} H_{66}\right) \text {, } \\
& b_{20}=\frac{c_{1}^{2}}{R_{2} a_{1} a_{2}} \frac{\partial a_{1}}{\partial y} H_{21} \\
& b_{21}=\frac{c_{1}^{2}}{R_{2} a_{1} a_{2}} \frac{\partial a_{1}}{\partial y} H_{66} \\
& b_{22}=\frac{c_{1}^{2}}{R_{2} a_{1} a_{2}} \frac{\partial a_{2}}{\partial x}\left(H_{22}-H_{66}\right) \text {, } \\
& b_{23}=\frac{1}{R_{2}}\left(A_{22}+A_{21}-\frac{c_{2}}{a_{2}} D_{44}+c_{3} a_{2} D_{44}-c_{2} c_{3} a_{2} F_{44}\right. \\
& a_{25}=\frac{c_{1}}{R_{1} a_{2}}\left(F_{66}-c_{1} H_{66}\right) \text {, } \\
& a_{26}=\frac{c_{1}}{R_{1}}\left(\frac{1}{a_{2}} F_{12}-\frac{c_{1}}{a_{2}} H_{12}+\frac{1}{a_{1}} F_{66}-\frac{c_{1}}{a_{1}} H_{66}\right), \\
& \left.+\frac{1}{a_{1}} A_{44}\right) \text {, } \\
& b_{24}=\frac{c_{1}}{R_{2} a_{2}}\left(F_{22}-c_{1} H_{22}\right) \text {, } \\
& a_{27}=\frac{c_{1}}{R_{1} a_{1} a_{2}} \frac{\partial a_{2}}{\partial x}\left(F_{12}-c_{1} H_{12}\right), \\
& a_{28}=\frac{c_{1}}{R_{1} a_{1} a_{2}} \frac{\partial a_{1}}{\partial y}\left(F_{11}-c_{1} H_{11}\right) \text {, } \\
& a_{29}=\frac{c_{1}}{R_{1} a_{1} a_{2}} \frac{\partial a_{1}}{\partial y}\left(-F_{66}+c_{1} H_{66}\right) \text {, } \\
& a_{30}=\frac{c_{1}}{R_{1} a_{1} a_{2}} \frac{\partial a_{2}}{\partial x}\left(-F_{66}+c_{1} H_{66}\right) \text {, } \\
& a_{31}=\frac{1}{R_{1}^{2}}\left(-A_{55}+c_{2} D_{55}\right) \text {, } \\
& a_{32}=\frac{1}{R_{1}}\left(A_{55}-c_{2} D_{55}+c_{3} a_{1} D_{55}-c_{2} c_{3} a_{1} F_{55}\right) \text {, } \\
& b_{11}=\frac{1}{a_{2}} A_{22} \text {, } \\
& b_{12}=\frac{1}{a_{1}} A_{66}, \\
& b_{13}=\left(\frac{1}{a_{1}} A_{21}+\frac{1}{a_{2}} A_{66}\right) \text {, } \\
& b_{14}=\frac{1}{a_{1} a_{2}} \frac{\partial a_{1}}{\partial y} A_{21} \text {, } \\
& b_{15}=\frac{1}{a_{1} a_{2}} \frac{\partial a_{2}}{\partial x} A_{22} \text {, } \\
& b_{16}=\frac{1}{a_{1} a_{2}} \frac{\partial a_{2}}{\partial x} A_{66} \text {, } \\
& b_{25}=\frac{c_{1}}{R_{2} a_{1}}\left(F_{66}-c_{1} H_{66}\right) \text {, } \\
& b_{26}=\frac{c_{1}}{R_{2}}\left(\frac{1}{a_{1}} F_{21}-\frac{c_{1}}{a_{1}} H_{21}+\frac{1}{a_{2}} F_{66}-\frac{c_{1}}{a_{2}} H_{66}\right) \text {, } \\
& b_{27}=\frac{c_{1}}{R_{2} a_{1} a_{2}} \frac{\partial a_{1}}{\partial y}\left(F_{21}-c_{1} H_{21}\right) \text {, } \\
& b_{28}=\frac{c_{1}}{R_{2} a_{1} a_{2}} \frac{\partial a_{2}}{\partial x}\left(F_{22}-c_{1} H_{22}\right) \text {, } \\
& b_{29}=\frac{c_{1}}{R_{2} a_{1} a_{2}} \frac{\partial a_{2}}{\partial x}\left(-F_{66}+c_{1} H_{66}\right) \text {, } \\
& b_{30}=\frac{c_{1}}{R_{2} a_{1} a_{2}} \frac{\partial a_{1}}{\partial y}\left(-F_{66}+c_{1} H_{66}\right), \\
& b_{31}=\frac{1}{R_{2}^{2}}\left(-A_{44}+c_{2} D_{44}\right) \text {, } \\
& b_{32}=\frac{1}{R_{2}}\left(A_{44}-c_{2} D_{44}+c_{3} a_{2} D_{44}-c_{2} c_{3} a_{2} F_{44}\right) \text {, } \\
& c_{11}=\frac{c_{1}^{3}}{a_{1}} H_{11}, \\
& c_{12}=\frac{c_{1}^{3}}{a_{2}} H_{22}, \\
& c_{13}=\frac{c_{1}^{2}}{a_{1} a_{2}} \frac{\partial a_{2}}{\partial x} H_{12}, \\
& c_{14}=\frac{c_{1}^{2}}{a_{1} a_{2}} \frac{\partial a_{1}}{\partial y} H_{21} \text {, }
\end{aligned}
$$




$$
\begin{aligned}
& c_{15}=\left(\frac{c_{1}^{3}}{a_{2}} H_{12}+\frac{c_{1}^{2}}{a_{1}} H_{66}+\frac{c_{1}^{2}}{a_{2}} H_{66}+\frac{c_{1}^{3}}{a_{1}} H_{21}\right), \\
& c_{36}=\frac{2}{a_{2}} A_{66}, \\
& c_{16}=\frac{c_{1}^{2}}{a_{1} a_{2}} \frac{\partial a_{1}}{\partial y}\left(-H_{11}+2 H_{66}\right) \text {, } \\
& c_{37}=\left(\frac{1}{a_{2}} A_{66}+\frac{1}{a_{1}} A_{21}\right), \\
& c_{17}=\frac{c_{1}^{2}}{a_{1} a_{2}} \frac{\partial a_{2}}{\partial x}\left(-H_{22}+2 H_{66}\right) \text {, } \\
& c_{38}=\frac{2}{a_{1} a_{2}} \frac{\partial a_{2}}{\partial x} A_{66}, \\
& c_{18}=\left(\frac{1}{R_{1}} A_{11}+\frac{1}{R_{2}} A_{12}\right) \text {, } \\
& c_{19}=\left(-\frac{c_{2}}{a_{1}} D_{55}-c_{2} c_{3} a_{1} F_{55}+\frac{1}{a_{1}} A_{55}+c_{3} a_{1} D_{55}\right) \text {, } \\
& c_{39}=\frac{1}{a_{1} a_{2}} \frac{\partial a_{2}}{\partial x} A_{66} \text {, } \\
& c_{40}=\left(\frac{1}{a_{1}} A_{66}+\frac{1}{a_{2}} A_{12}\right), \\
& c_{20}=\left(\frac{1}{R_{1}} A_{21}+\frac{1}{R_{2}} A_{22}\right) \text {, } \\
& c_{21}=\left(-\frac{c_{2}}{a_{2}} D_{44}-c_{2} c_{3} a_{2} F_{44}+\frac{1}{a_{2}} A_{44}+c_{3} a_{2} D_{44}\right) \text {, } \\
& c_{41}=\frac{1}{a_{1} a_{2}} \frac{\partial a_{1}}{\partial y} A_{66} \text {, } \\
& c_{42}=\frac{1}{a_{2}} A_{66}, \\
& c_{43}=\frac{1}{a_{1} a_{2}} \frac{\partial a_{2}}{\partial x} A_{66}, \\
& c_{44}=\frac{1}{a_{1}} A_{21} \text {, } \\
& c_{45}=\frac{1}{a_{1} a_{2}} \frac{\partial a_{1}}{\partial y} A_{21}, \\
& c_{46}=\frac{1}{a_{1} a_{2}} \frac{\partial a_{1}}{\partial y} A_{21}, \\
& c_{47}=\left(\frac{1}{R_{1}} A_{21}+\frac{1}{R_{2}} A_{22}\right) \text {, } \\
& c_{48}=\frac{1}{a_{2}} A_{22} \text {, } \\
& c_{49}=\frac{1}{a_{2}} A_{22} \text {, } \\
& c_{50}=\frac{1}{a_{1} a_{2}} \frac{\partial a_{2}}{\partial x} A_{22}, \\
& c_{51}=\frac{1}{a_{1} a_{2}} \frac{\partial a_{2}}{\partial x} A_{22}, \\
& c_{52}=\left(\frac{c_{1}}{a_{1}} F_{11}-\frac{c_{1}^{2}}{a_{1}} H_{11}\right) \text {, } \\
& c_{53}=\left(\frac{c_{1}}{a_{2}} F_{22}-\frac{c_{1}^{2}}{a_{2}} H_{22}\right) \text {, } \\
& c_{32}=\frac{2}{a_{1}} A_{66} \text {, } \\
& c_{33}=\frac{1}{a_{1}} A_{66}, \\
& c_{34}=\frac{2}{a_{1} a_{2}} \frac{\partial a_{1}}{\partial y} A_{66} \text {, } \\
& c_{54}=\left(\frac{c_{1}}{a_{2}} F_{12}-\frac{c_{1}^{2}}{a_{2}} H_{12}+\frac{2 c_{1}}{a_{1}} F_{66}-\frac{2 c_{1}^{2}}{a_{1}} H_{66}\right) \text {, } \\
& c_{55}=\left(\frac{c_{1}}{a_{1}} F_{21}-\frac{c_{1}^{2}}{a_{1}} H_{21}+\frac{2 c_{1}}{a_{2}} F_{66}-\frac{2 c_{1}^{2}}{a_{2}} H_{66}\right) \text {, } \\
& c_{35}=\frac{1}{a_{1} a_{2}} \frac{\partial a_{1}}{\partial y} A_{66}, \\
& c_{56}=\frac{c_{1}}{a_{1} a_{2}} \frac{\partial a_{1}}{\partial y}\left(F_{11}-c_{1} H_{11}\right) \text {, }
\end{aligned}
$$




$$
\begin{aligned}
& c_{57}=\frac{c_{1}}{a_{1} a_{2}} \frac{\partial a_{2}}{\partial x}\left(F_{12}-c_{1} H_{12}\right), \\
& d_{22}=\frac{c_{1}}{a_{1} a_{2}} \frac{\partial a_{1}}{\partial y}\left(-F_{11}+F_{66}+c_{1} H_{11}-c_{1} H_{66}\right) \text {, } \\
& c_{58}=\frac{c_{1}}{a_{1} a_{2}} \frac{\partial a_{1}}{\partial y}\left(F_{21}-c_{1} H_{21}\right) \text {, } \\
& d_{23}=\left(\frac{c_{2}}{a_{1}} D_{55}+c_{2} c_{3} a_{1} F_{55}-\frac{1}{a_{1}} A_{55}-c_{3} a_{1} D_{55}\right) \text {, } \\
& c_{59}=\frac{c_{1}}{a_{1} a_{2}} \frac{\partial a_{2}}{\partial x}\left(F_{22}-c_{1} H_{22}\right) \text {, } \\
& c_{60}=\frac{2 c_{1}}{a_{1} a_{2}} \frac{\partial a_{1}}{\partial y}\left(-F_{66}+c_{1} H_{66}\right) \text {, } \\
& c_{61}=\frac{2 c_{1}}{a_{1} a_{2}} \frac{\partial a_{2}}{\partial x}\left(F_{66}-c_{1} H_{66}\right) \text {, } \\
& c_{62}=\frac{1}{R_{1} a_{1} a_{2}} \frac{\partial a_{2}}{\partial x}\left(A_{12}+A_{22}\right) \text {, } \\
& c_{63}=\frac{1}{R_{1} a_{1} a_{2}} \frac{\partial a_{1}}{\partial y}\left(A_{11}+A_{21}\right) \text {, } \\
& c_{64}=\left(\frac{1}{R_{1}^{2}} A_{11}+\frac{1}{R_{1} R_{2}} A_{12}+\frac{1}{R_{1} R_{2}} A_{21}+\frac{1}{R_{2}^{2}} A_{22}\right) \text {, } \\
& d_{11}=\frac{1}{a_{1}}\left(D_{11}-2 c_{1} F_{11}+c_{1}^{2} H_{11}\right) \text {, } \\
& d_{12}=\frac{1}{a_{2}}\left(D_{66}-2 c_{1} F_{66}+c_{1}^{2} H_{66}\right) \text {, } \\
& d_{13}=\frac{1}{a_{1} a_{2}} \frac{\partial a_{2}}{\partial x}\left(D_{12}-2 c_{1} F_{12}+c_{1}^{2} H_{12}\right) \text {, } \\
& d_{14}=\frac{1}{a_{1} a_{2}} \frac{\partial a_{1}}{\partial y}\left(-D_{66}+2 c_{1} F_{66}-c_{1}^{2} H_{66}\right) \text {, } \\
& d_{15}=\frac{1}{a_{1} a_{2}} \frac{\partial a_{1}}{\partial y}\left(D_{11}-2 c_{1} F_{11}+c_{1}^{2} H_{11}\right) \text {, } \\
& d_{16}=\frac{1}{a_{1} a_{2}} \frac{\partial a_{2}}{\partial x}\left(-D_{66}+2 c_{1} F_{66}-c_{1}^{2} H_{66}\right) \text {, } \\
& d_{17}=\left(\frac{1}{a_{2}} D_{12}+\frac{1}{a_{1}} D_{66}-\frac{2 c_{1}}{a_{2}} F_{12}+\frac{c_{1}^{2}}{a_{2}} H_{12}\right. \\
& \left.+\frac{c_{1}^{2}}{a_{1}} H_{66}\right) \\
& d_{18}=\frac{c_{1}}{a_{1}}\left(-F_{11}+c_{1} H_{11}\right) \text {, } \\
& d_{19}=-\frac{c_{1}}{a_{2}} F_{12}-\frac{c_{1}}{a_{1}} F_{66}-\frac{c_{1}}{a_{2}} F_{66}+\frac{c_{1}^{2}}{a_{2}} H_{12}+\frac{c_{1}^{2}}{a_{1}} H_{66} \\
& +\frac{c_{1}^{2}}{a_{2}} H_{66} \\
& d_{20}=\frac{c_{1}}{a_{1} a_{2}} \frac{\partial a_{2}}{\partial x}\left(-F_{12}+c_{1} H_{12}\right), \\
& d_{21}=\frac{c_{1}}{a_{1} a_{2}} \frac{\partial a_{2}}{\partial x}\left(F_{66}-c_{1} H_{66}\right) \text {, } \\
& d_{24}=\frac{1}{R_{1}}\left(-c_{2} D_{55}+A_{55}\right) \text {, } \\
& d_{25}=\left(c_{2} D_{55}-A_{55}+c_{2} c_{3} a_{1} F_{55}-c_{3} a_{1} D_{55}\right) \text {, } \\
& e_{11}=\frac{1}{a_{2}}\left(D_{22}-2 c_{1} F_{22}+c_{1}^{2} H_{22}\right) \text {, } \\
& e_{12}=\frac{1}{a_{1}}\left(D_{66}-2 c_{1} F_{66}+c_{1}^{2} H_{66}\right) \text {, } \\
& e_{13}=\frac{1}{a_{1} a_{2}} \frac{\partial a_{1}}{\partial y}\left(D_{21}-2 c_{1} F_{21}+c_{1}^{2} H_{21}\right) \text {, } \\
& e_{14}=\frac{1}{a_{1} a_{2}} \frac{\partial a_{2}}{\partial x}\left(-D_{66}+2 c_{1} F_{66}-c_{1}^{2} H_{66}\right) \text {, } \\
& e_{15}=\frac{1}{a_{1} a_{2}} \frac{\partial a_{2}}{\partial x}\left(D_{22}-2 c_{1} F_{22}+c_{1}^{2} H_{22}\right) \text {, } \\
& e_{16}=\frac{1}{a_{1} a_{2}} \frac{\partial a_{1}}{\partial y}\left(-D_{66}+2 c_{1} F_{66}-c_{1}^{2} H_{66}\right) \text {, } \\
& e_{17}=\left(\frac{1}{a_{1}} D_{21}+\frac{1}{a_{2}} D_{66}-\frac{2 c_{1}}{a_{1}} F_{21}+\frac{c_{1}^{2}}{a_{1}} H_{21}\right. \\
& \left.+\frac{c_{1}^{2}}{a_{2}} H_{66}\right) \\
& e_{18}=\frac{c_{1}}{a_{2}}\left(-F_{22}+c_{1} H_{22}\right) \text {, } \\
& e_{19}=-\frac{c_{1}}{a_{1}} F_{21}-\frac{c_{1}}{a_{2}} F_{66}-\frac{c_{1}}{a_{1}} F_{66}+\frac{c_{1}^{2}}{a_{1}} H_{21}+\frac{c_{1}^{2}}{a_{2}} H_{66} \\
& +\frac{c_{1}^{2}}{a_{1}} H_{66} \\
& e_{20}=\frac{c_{1}}{a_{1} a_{2}} \frac{\partial a_{1}}{\partial y}\left(-F_{21}+c_{1} H_{21}\right) \text {, } \\
& e_{21}=\frac{c_{1}}{a_{1} a_{2}} \frac{\partial a_{1}}{\partial y}\left(F_{66}-c_{1} H_{66}\right) \text {, } \\
& e_{22}=\frac{c_{1}}{a_{1} a_{2}} \frac{\partial a_{2}}{\partial x}\left(-F_{22}+F_{66}+c_{1} H_{22}-c_{1} H_{66}\right) \text {, } \\
& e_{23}=\left(\frac{c_{2}}{a_{2}} D_{44}+c_{2} c_{3} a_{2} F_{44}-\frac{1}{a_{2}} A_{44}-c_{3} a_{2} D_{44}\right) \text {, } \\
& e_{24}=\frac{1}{R_{2}}\left(-c_{2} D_{44}+A_{44}\right) \text {, } \\
& e_{25}=\left(c_{2} D_{44}-A_{44}+c_{2} c_{3} a_{2} F_{44}-c_{3} a_{2} D_{44}\right) \text {. }
\end{aligned}
$$




\section{Conflicts of Interest}

The authors declare that they have no conflicts of interest.

\section{Acknowledgments}

The authors gratefully acknowledge the support of the National Natural Science Foundation of China (NNSFC) through Grant nos. 11572006, 11202009, 11072008, and 10732020 and the Funding Project for Academic Human Resources Development in Institutions of Higher Learning under the Jurisdiction of Beijing Municipality (PHRIHLB).

\section{References}

[1] W. K. Wilkie, R. G. Bryant, J. W. High et al., "Low-cost piezocomposite actuator for structural control applications," in Proceeding of the SPIE's 7th Annual International Symposium on Smart Structures and Materials, vol. 3991, 2000.

[2] R. B. Williams, B. W. Grimsley, D. J. Inman, and W. K. Wilkie, "Manufacturing and mechanics-based characterization of macro fiber composite actuators," in Proceeding of the ASME 2002 International Mechanical Engineering Congress and Exposition, pp. 79-89, 2002.

[3] S.-C. Choi, J.-S. Park, and J.-H. Kim, "Vibration control of pretwisted rotating composite thin-walled beams with piezoelectric fiber composites," Journal of Sound and Vibration, vol. 300, no. 1-2, pp. 176-196, 2007.

[4] H. P. Konka, M. A. Wahab, and K. Lian, "Piezoelectric fiber composite transducers for health monitoring in composite structures," Sensors and Actuator, A: Physical, vol. 194, no. 5, pp. 84-94, 2013.

[5] A. Y. Tamijani, M. Abouhamze, R. Mirzaeifar, A. R. Ohadi, and M. R. Eslami, "Feedback control of piezo-laminate composite plate," in Proceeding of the 14th International Congress on Sound and Vibration 2007, (ICSV '07), vol. 7, pp. 9-12, Cairns, Australia, 2007.

[6] S. K. Parashar, A. DasGupta, U. Von Wagner, and P. Hagedorn, "Non-linear shear vibrations of piezoceramic actuators," International Journal of Non-Linear Mechanics, vol. 40, no. 4, pp. 429-443, 2005.

[7] X.-K. Xia and H.-S. Shen, "Nonlinear vibration and dynamic response of FGM plates with piezoelectric fiber reinforced composite actuators," Composite Structures, vol. 90, no. 2, pp. 254-262, 2009.

[8] S. Raja, G. Prathap, and P. K. Sinha, "Active vibration control of composite sandwich beams with piezoelectric extensionbending and shear actuators," Smart Materials and Structures, vol. 11, no. 1, pp. 63-71, 2002.

[9] D. Thakkar and R. Ganguli, "Helicopter vibration reduction in forward flight with induced-shear based piezoceramic actuation," Smart Materials and Structures, vol. 13, no. 3, pp. 599-608, 2004.

[10] M. A. Trindade and T. Y. Kakazu, "Structural control of sandwich beams using shear piezoelectric actuators subjected to large electric fields," in Proceedings of the 4th ABCM National Congress of Mechanical Engineering (CONEM '06), Recife, Brazil, 2006.

[11] H. Y. Zhang and Y. P. Shen, "Vibration suppression of laminated plates with 1-3 piezoelectric fiber-reinforced composite layers equipped with interdigitated electrodes," Composite Structures, vol. 79, no. 2, pp. 220-228, 2007.

[12] R. B. Williams, D. J. Inman, and W. K. Wilkie, "Nonlinear response of the macro fiber composite actuator to monotonically increasing excitation voltage," Journal of Intelligent Material Systems and Structures, vol. 17, no. 7, pp. 601-608, 2006.

[13] M. S. Azzouz and C. Hall, "Nonlinear finite element analysis of a rotating MFC actuator," in Proceeding of the 51st AIAA/ASME/ASCE/AHS/ASC Structures, Structural Dynamics and Materials Conference, Orlando, Fla, USA, 2010.

[14] M. A. Trindade and A. Benjeddou, "Finite element characterization and parametric analysis of the nonlinear behaviour of an actual d15 shear MFC," Acta Mechanica, vol. 224, no. 11, pp. 2489-2503, 2013.

[15] K. Steiger and P. Mokry, "Finite element analysis of the macro fiber composite ctuator: macroscopic elastic and piezoelectric properties and active control thereof by means of negative capacitance shunt circuit," Smart Materials \&amp; Structures, vol. 24, pp. 025-026, 2015.

[16] S.-Q. Zhang, Y.-X. Li, and R. Schmidt, "Modeling and simulation of macro-fiber composite layered smart structures," Composite Structures, vol. 126, pp. 89-100, 2015.

[17] S. Gohari, S. Sharifi, and Z. Vrcelj, "New explicit solution for static shape control of smart laminated cantilever piezo-composite-hybrid plates/beams under thermo-electromechanical loads using piezoelectric actuators," Composite Structures, vol. 145, pp. 89-112, 2016.

[18] J.-S. Park and J.-H. Kim, "Suppression of aero-thermal large deflections and snap-through behaviors of composite panels using macro fiber composite actuators," Smart Materials and Structures, vol. 13, no. 6, pp. 1448-1459, 2004.

[19] H. S. Kim, J. W. Sohn, and S.-B. Choi, "Vibration control of a cylindrical shell structure using macro fiber composite actuators," Mechanics Based Design of Structures and Machines, vol. 39, no. 4, pp. 491-506, 2011.

[20] M.-L. Dano and B. Jullière, "Active control of thermally induced distortion in composite structures using Macro Fiber Composite actuators," Smart Materials and Structures, vol. 16, no. 6, pp. 2315-2322, 2007.

[21] M. H. Korayem and A. Homayooni, "The size-dependent analysis of multilayer micro-cantilever plate with piezoelectric layer incorporated voltage effect based on a modified couple stress theory," European Journal of Mechanics. A. Solids, vol. 61, pp. 59-72, 2017.

[22] J. Q. Li, Z. R. Ma, Z. H. Wang, and Y. Narita, "Random vibration control of laminated composite plates with piezoelectric fiber reinforced composites," Acta Mechanica Solida Sinica, vol. 29, no. 3, pp. 316-327, 2016.

[23] R. Suresh Kumar and M. C. Ray, "Active control of geometrically nonlinear vibrations of doubly curved smart sandwich shells using 1-3 piezoelectric composites," Composite Structures, vol. 105, pp. 173-187, 2013.

[24] Y. Sapsathiarn, T. Senjuntichai, and R. K. N. D. Rajapakse, "Electro-mechanical load transfer from a fiber in a 1-3 piezocomposite with an imperfect interface," Composites Part B: Engineering, vol. 39, no. 7-8, pp. 1114-1124, 2008.

[25] A. Deraemaeker and H. Nasser, "Numerical evaluation of the equivalent properties of Macro Fiber Composite (MFC) transducers using periodic homogenization," International Journal of Solids and Structures, vol. 47, no. 24, pp. 3272-3285, 2010.

[26] I. V. Andrianov, V. V. Danishevs'kyy, and A. L. Kalamkarov, "Micromechanical analysis of fiber-reinforced composites on 
account of influence of fiber coatings," Composites Part B: Engineering, vol. 39, no. 5, pp. 874-881, 2008.

[27] F. Biscani, H. Nasser, S. Belouettar, and E. Carrera, "Equivalent electro-elastic properties of Macro Fiber Composite (MFC) transducers using asymptotic expansion approach," Composites Part B: Engineering, vol. 42, no. 3, pp. 444-455, 2011.

[28] S. S. Prasath and A. Arockiarajan, "Analytical, numerical and experimental predictions of the effective electromechanical properties of macro-fiber composite (MFC)," Sensors and Actuators, vol. A214, pp. 31-44, 2014.

[29] S. S. Prasath and A. Arockiarajan, "Experimental and theoretical investigation on the thermo-electro-elastic properties of Macro-Fiber Composites (MFC)," Composite Structures, vol. 122, pp. 8-22, 2015.

[30] J. Plattenburg, J. T. Dreyer, and R. Singh, "Active and passive damping patches on a thin rectangular plate: a refined analytical model with experimental validation," Journal of Sound and Vibration, vol. 353, pp. 75-95, 2015.

[31] A. Pandey and A. Arockiarajan, "Actuation performance of macro-fiber composite (MFC): modeling and experimental studies,' Sensors and Actuator, A: Physical, vol. 248, pp. 114-129, 2016.

[32] T. Kashiwao, I. Izadgoshasb, Y. Y. Lim, and M. Deguchi, "Optimization of rectifier circuits for a vibration energy harvesting system using a macro-fiber composite piezoelectric element," Microelectronics Journal, vol. 54, pp. 109-115, 2016. 

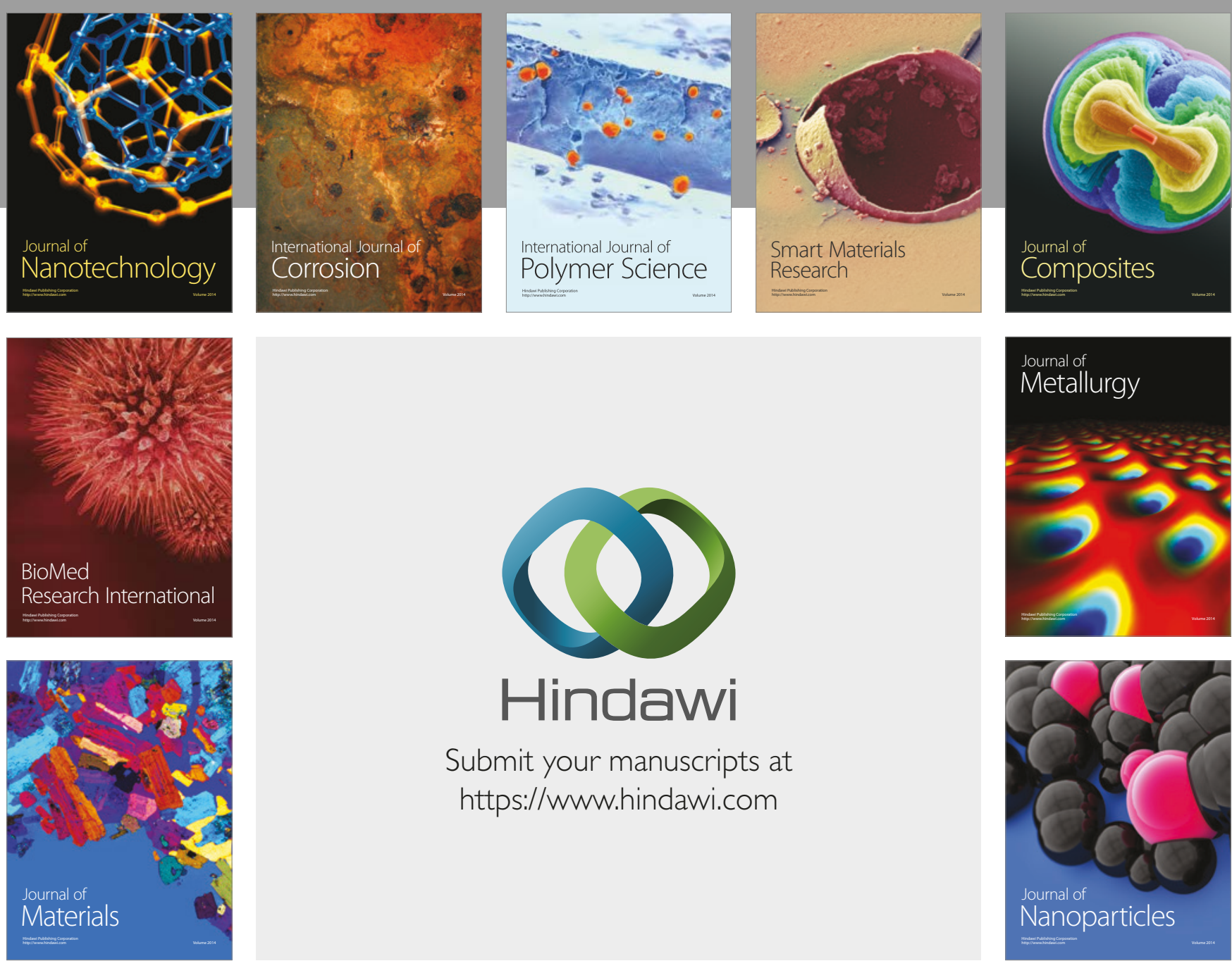

\section{Hindawi}

Submit your manuscripts at

https://www.hindawi.com
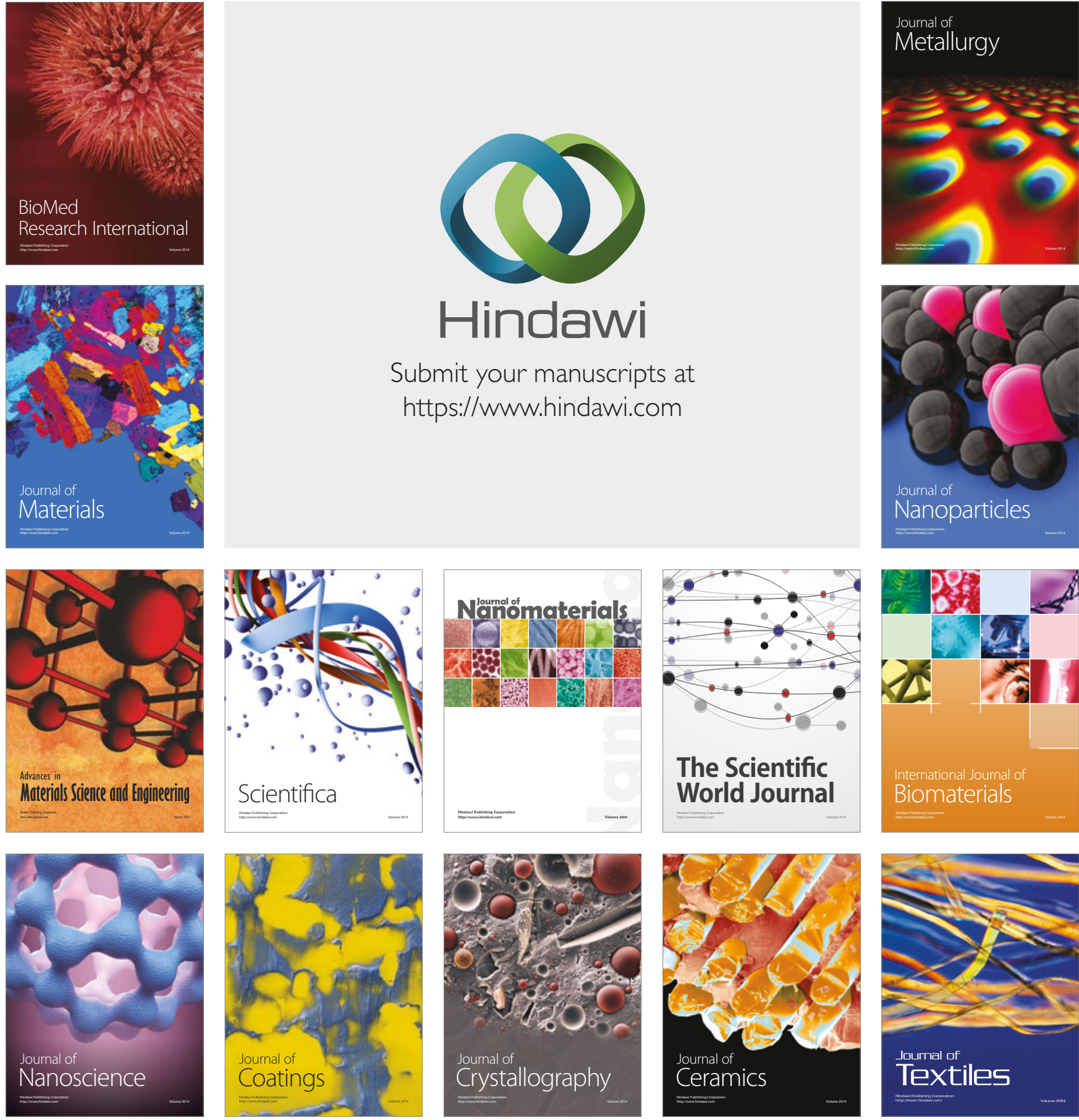

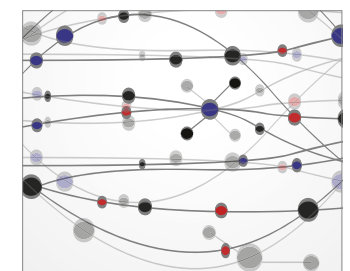

The Scientific World Journal
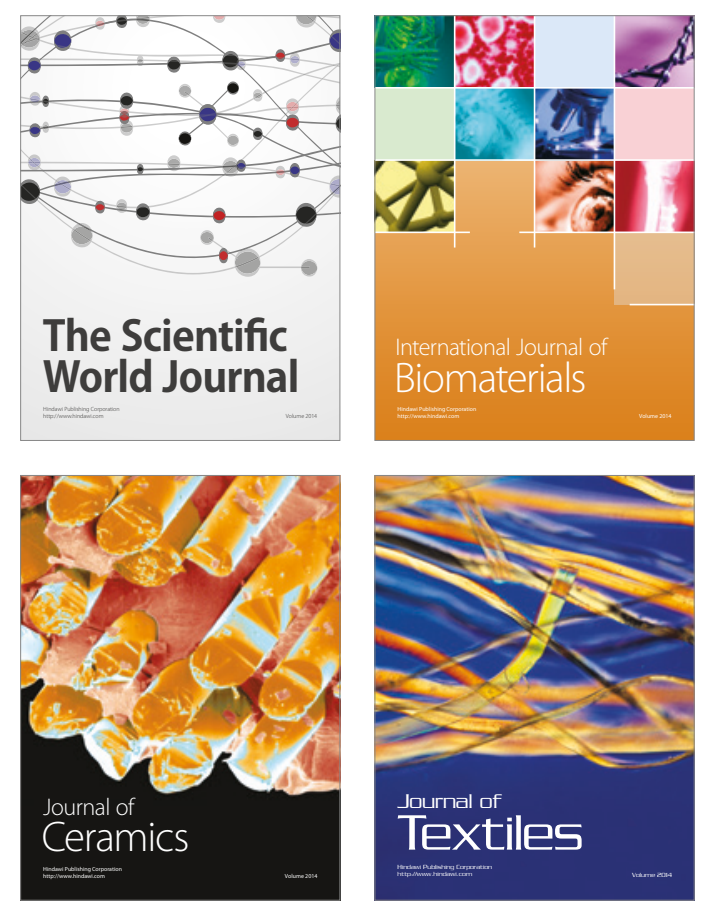